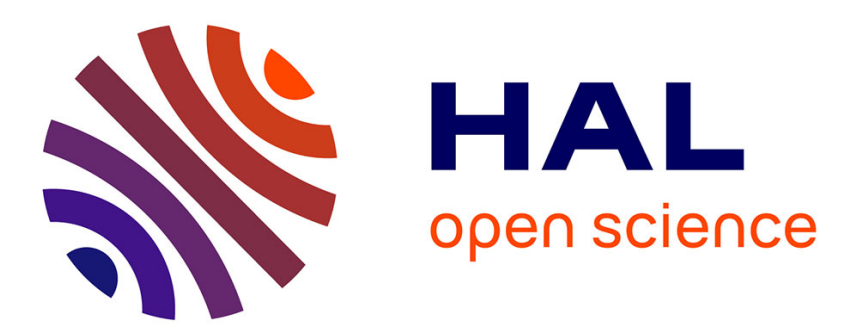

\title{
Microfluidic Whispering Gallery Mode Optical Sensors for Biological Applications
}

Yuye Wang, Shuwen Zeng, Georges Humbert, Ho-Pui Ho

\section{To cite this version:}

Yuye Wang, Shuwen Zeng, Georges Humbert, Ho-Pui Ho. Microfluidic Whispering Gallery Mode Optical Sensors for Biological Applications. Laser and Photonics Reviews, 2020, 10.1002/lpor.202000135 . hal-03023104

\section{HAL Id: hal-03023104 \\ https://hal-unilim.archives-ouvertes.fr/hal-03023104}

Submitted on 25 Nov 2020

HAL is a multi-disciplinary open access archive for the deposit and dissemination of scientific research documents, whether they are published or not. The documents may come from teaching and research institutions in France or abroad, or from public or private research centers.
L'archive ouverte pluridisciplinaire HAL, est destinée au dépôt et à la diffusion de documents scientifiques de niveau recherche, publiés ou non, émanant des établissements d'enseignement et de recherche français ou étrangers, des laboratoires publics ou privés. 


\section{WILEY-VCH}

\section{Microfluidic Whispering Gallery Mode Optical Sensors for Biological Applications}

Yuye Wang, Shuwen Zeng ${ }^{*}$, Georges Humbert, Ho-Pui Ho ${ }^{*}$

Abstract: Whispering gallery mode (WGM) resonators have been exploited as a highly sensitive and efficient sensing technique in the past few years. They offer the advantages of ultra-high sensitivity, compact size and label-free sensing capability. More recently, with the rapid development of microfluidic technologies, many integrated WGM sensors, by combining the portability of lab-on-chip devices and the high sensitivity of WGM resonators, have emerged. The capabilities of efficient sample handling and multiplexed analyte detection offered by these systems have led to many biological and chemical sensing applications, especially for the detection of single particle or biomolecule. In this review, different sensing mechanisms based on integrated whispering gallery mode resonators are introduced. Various geometries of WGM cavities including solid, liquid and hollow resonators and a detailed discussion on their integration with microfluidic devices are also covered. Different types of packaging schemes and integration methods are compared in terms of ease of fabrication and device performance. Finally, recent applications of microfluidic-based WGM sensors for detecting biological and chemical target molecules are discussed, with a prospect on future challenges and trends of development in microfluidics for multiplexed detection.

Keywords: Integrated sensors; Optical micro resonators; Optofluidics; Multiplexed detection; Label-free optical sensing. 


\section{WILEY-VCH}

\section{Introduction}

Optical sensors are known to be a useful analytical tool in various fields of bio-chemical detection ${ }^{[1,2]}$ including disease screening ${ }^{[3,4]}$, environmental monitoring ${ }^{[5-7]}$ and food security ${ }^{[8,9]}$. They are also promising tools for clinical diagnostics ${ }^{[10]}$. The optical signals collected in terms of light intensity, frequency, phase and polarization provide the corresponding information such as the gas/liquid concentration, pressure, temperature and other characteristics of the detected objects. Over the past decade, there is an increasing research development on label-free techniques for real-time sensing due to the need for in-situ and portable devices. Nowadays, several label-free optical sensing techniques have been investigated and welldeveloped, e.g., whispering gallery mode (WGM) resonators, surface plasmon resonance ${ }^{[11]}$, Mach-Zehnder interferometers ${ }^{[12]}$, photonic crystal cavities ${ }^{[13]}$ and photonic bragg fibers ${ }^{[14]}$. These sensing devices are free of bio-recognition and signal amplification element such as fluorescent tags, which makes no contamination to the analytes themselves and enables highthroughput screening ${ }^{[15]}$.

Among them, WGM resonators possess a relatively high quality factor (Q), which is generally defined as $Q=\lambda_{0} / \Delta \lambda$ (where $\lambda_{0}$ is the resonance wavelength and $\Delta \lambda$ is the linewidth of the optical resonance in the spectra), up to $10^{11[16]}$ based on the minimal reflection. The high Q value indicates a very narrow spectral linewidth (less than $100 \mathrm{kHz}$ at $1550 \mathrm{~nm})^{[17]}$ and a large circulating power (typically around $100 \mathrm{~W})^{[18]}$. WGM structures also have the advantages of small mode volume, rapid response speed and compatibility with aqueous environment ${ }^{[19]}$. As a result, WGM sensors have become a more and more powerful tool for biological and chemical applications over these years ${ }^{[20]}$. Extremely high sensitivities were recently presented in various application scenarios including temperature sensing (up to $0.96 \mathrm{~nm} /{ }^{\circ} \mathrm{C}$ ) ${ }^{[21]}$, humidity sensing (up to $47 \mathrm{pm} / \% \mathrm{RH}$ ) ${ }^{[22]}$, pressure sensing (up to $38 \mathrm{GHz} / \mathrm{bar}{ }^{[23]}$, and detection of single biomolecule such as exosome ${ }^{[24]}$, lentivirus ${ }^{[25]}$ or protein ${ }^{[26,27]}$. 


\section{WILEY-VCH}

Despite much progress in terms of sensitivity improvement, many of these WGM sensors only operate under sophisticated laboratory environment with complicated experimental procedures and bulky instruments ${ }^{[19,20,28-30]}$. The experimental setup is usually cumbersome, with large volumetric reagent consumption and long assay time, which makes them difficult to perform on-site, multiplexed and rapid sensing tests. For example, one of the major challenges in biomedical applications especially for disease diagnosis is the detection of multiple biomarkers with one single chip using minimal sample volume. Multiplexed detection is therefore extremely useful under these circumstances. It allows for parallel detection of multiple analytes, thus largely increasing the sample throughput and improving the sensing efficiency [31, 32]. Lab-on-a-chip (LOC) devices and microfluidic systems thus have become useful solutions to the above-mentioned challenges. They aim to manipulate liquids at micro- or even nano-liter scale, which largely decrease the sample volume and reduce the reagent cost. Furthermore, complicated sample handling processes (including separation, mixing and sorting) and multiple parallel measurements can be performed simply through the design of microchannels inside the fluidic-chip platform ${ }^{[31,33-35]}$. More importantly, the portability and robustness of LOC devices are highly desired in clinical applications and point-of-care diagnostics ${ }^{[36,37]}$. Therefore, there is a growing need in developing and engineering the microfluidic-based WGM sensors for portable and integrated devices ${ }^{[29]}$. Combining with microfluidics, the sequential loading of liquids, controllable reaction of sample solutions and multiplexed sensing with higher throughput and efficiency can be achieved simultaneously. Here, we provide a detailed discussion on the recent development on the combination of WGM sensors with microfluidic devices in this review, which is barely introduced in other literature.

Overall, this review will firstly focus on the basic sensing mechanism of WGM sensors, followed by introducing various geometries of WGM resonators, especially their recent development of integration with microfluidic LOC devices. Furthermore, the advantages and 


\section{WILEY-VCH}

practical examples of integrated WGM sensors and their sensing applications in various areas will be described. Lastly, we will also discuss the future challenges and perspectives of integrated WGM sensors.

\section{Sensing mechanism for optical WGM sensors}

Optical whispering gallery mode (WGM) is a type of electromagnetic wave that travels inside a resonator due to consecutive total internal reflection. While they are confined within the resonator, some energy of WGM will extend to the surrounding medium and form an evanescent field. The fluctuations in this evanescent field will result in the modification of optical path ${ }^{[38]}$, the shortening of cavity lifetime ${ }^{[25]}$ or the crosstalk between two counterpropagating modes ${ }^{[39,40]}$, thus causing detectable changes in WGM modes ${ }^{[29]}$. As a result, WGMs serve as excellent sensors to detect the perturbations in the surrounding environment caused by temperature, humidity, bio-reactions, etc.

\subsection{Basic sensing mechanism}

According to the type of changes in modes, we can generally divide the sensing mechanism into three categories ${ }^{[1,20,29,30,41]}$ : Mode-shift based sensing, mode-broadening based sensing and mode-splitting based sensing, respectively.

\subsubsection{Mode-shift based sensing}

Mode-shift based sensing is one of the most commonly adopted mechanisms in WGM sensors. When particles or analytes interact with the evanescent field of the resonator, a shift in resonance frequency will take place ${ }^{[42]}$. A simple illustration is depicted in Figure 1. This process can be explained by the reactive sensing principle. When a particle is bound to the 


\section{WILEY-VCH}

resonator, it will pull a part of the optical field outward, leading to an increase in the optical path, which further results in the mode shift. According to the first order perturbation theory, the angular frequency shift can be determined by the following equation ${ }^{[43,44]}$ :

$$
\frac{\Delta \omega}{\omega_{0}} \cong-\frac{\alpha_{e x}\left|E\left(r_{d}\right)\right|^{2}}{2 \int \delta(r)|E(r)|^{2} d V_{m}}
$$

where $\omega$ represents the angular frequency, $\alpha_{e x}$ is the polarization of the particle, $E(r)$ is the electric field throughout the WGM resonator while $E\left(r_{d}\right)$ is the electric field at specific particle position $r_{d}, \delta(r)$ is the permittivity and $V_{m}$ is the mode volume. According to this equation, increasing the proportion of the electric field that interacts with the detected object is an effective way to enhance the sensitivity.

Mode-shift based sensing is capable of measuring a wide range of parameters such as refractive index ${ }^{[45]}$, absorption of analytes ${ }^{[46,47]}$ and many physical features of the surrounding environment ${ }^{[48-50]}$ including temperature, humidity, pressure, etc. Versatile as it is, it also brings some problems in terms of accuracy and specificity since too many variables can result in the frequency response. 


\section{WILEY-VCH}

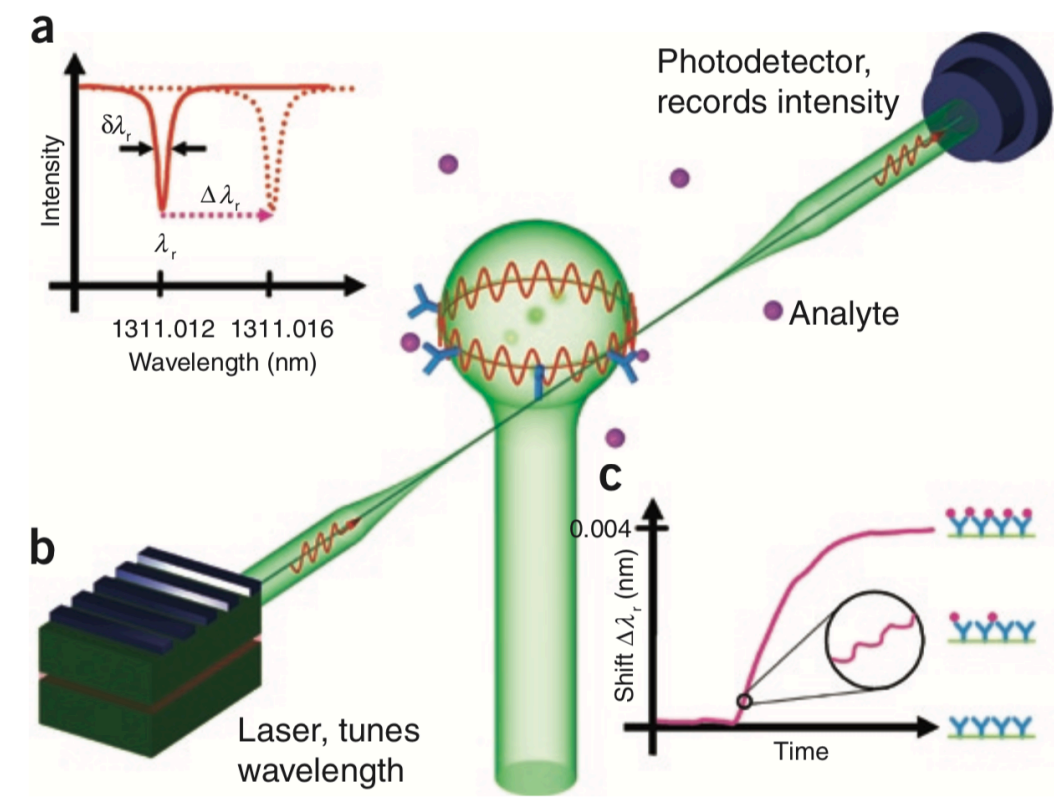

Figure 1. Mode-shift based sensing (a) Transmission spectra showing the mode shift. (b) Schematic of the basic WGM sensor setup. (c) Resonance wavelength shift in analyte binding process. Reproduced from ref. ${ }^{[42]}$. Copyright 2008 Springer Nature.

\subsubsection{Mode-broadening based sensing}

The linewidth of WGM indicates the energy loss per light wave oscillation ${ }^{[29]}$. In sensing applications, the energy loss usually increases when the analytes enter the evanescent field, thus broadening the linewidth. The broadening of linewidth results from both absorption loss $\left(\Delta \gamma_{a b s}\right)$ and scattering loss $\left(\Delta \gamma_{s c a}\right)$, which can be calculated through the following equations ${ }^{[51]}$ respectively:

$$
\begin{gathered}
\Delta \gamma_{a b s} \cong-\frac{\operatorname{Im}\left[\alpha_{e x}\right]\left|E\left(r_{d}\right)\right|^{2} \omega_{0}}{\int \delta(r)|E(r)|^{2} d V_{m}} \\
\Delta \gamma_{s c a} \cong-\frac{\omega_{0}^{4} n_{m}{ }^{5} \varepsilon_{0}}{6 \pi c^{3}} \frac{\left|\alpha_{e x}\right|^{2}\left|E\left(r_{d}\right)\right|^{2}}{\int \delta(r)|E(r)|^{2} d V_{m}}
\end{gathered}
$$




\section{WILEY-VCH}

where $\varepsilon_{0}$ is the permittivity in vacuum, $\mathrm{n}_{\mathrm{m}}$ is the refractive index of the WGM resonator while $\mathrm{c}$ is the speed of light in vacuum.

Figure 2 shows an example of mode-broadening based sensing. Different from mode-shift based sensing, mode-broadening based sensing is not sensitive to the changes of ambient conditions, which allows for the sensing of small particles with higher specificity and accuracy ${ }^{[25]}$. In this system, the detection of an individual nanoparticle with a radius of $70 \mathrm{~nm}$ and a single virus have been realized. However, since mode-broadening based sensing is essentially based on the energy loss of the cavity, it will no longer function if the analytes are attached on the WGM resonator for a consistent period of time ${ }^{[52,53]}$.

(a)

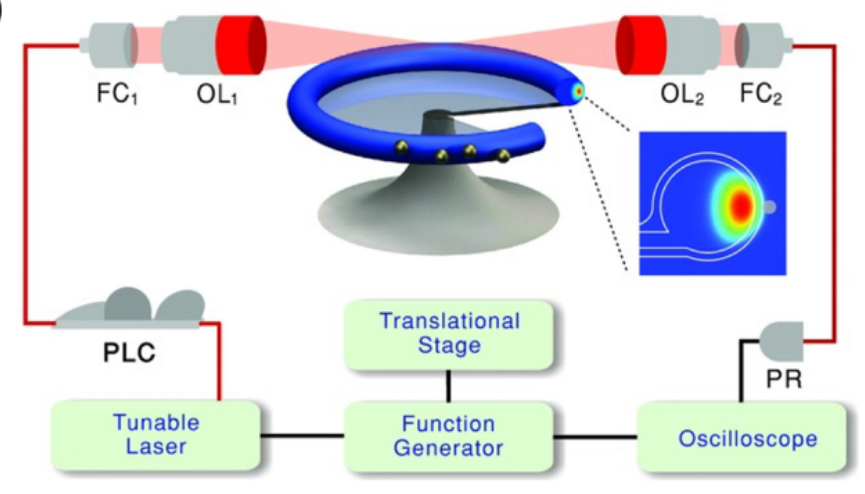

(b)

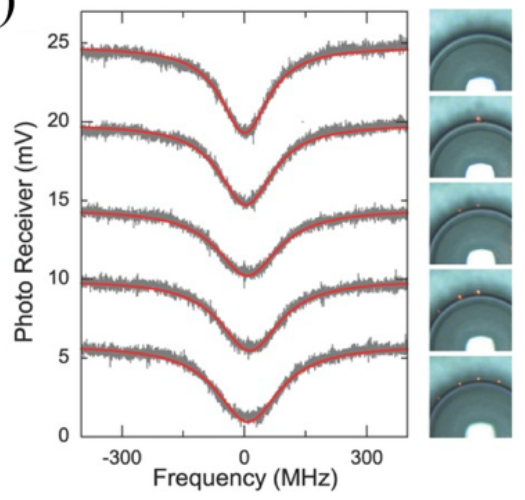

Figure 2. Mode-broadening based sensing (a) Experimental setup for mode-broadening based PS nanoparticles and virus detection. (b) Transmission spectra of the mode and the corresponding microcavityenhanced-scattering optical images upon binding of 0 - 4 nanoparticles (from top to bottom). Reproduced from ref. ${ }^{[25]}$. Copyright 2013 John Wiley and Sons.

\subsubsection{Mode-splitting based sensing}

In WGM resonators, the optical modes possess a two-fold degeneracy since light can propagate in both clockwise $(\mathrm{CW})$ and counterclockwise $(\mathrm{CCW})$ directions ${ }^{[54]}$. These counterpropagating degenerate modes share the same mode characteristics including resonant 


\section{WILEY-VCH}

frequency and field distributions. When a particle or molecule is bound to the surface of the WGM resonator, part of the light is scattered into the surrounding environment while the rest of it will be scattered back from the original propagating direction $(\mathrm{CW}, \mathrm{CCW})$ to the opposite one $(\mathrm{CCW}, \mathrm{CW})$, thus inducing the coupling between the two modes ${ }^{[55]}$ and further lifting the degeneracy between them. Two standing wave modes (SWMs) will form due to the resulting interference between these modes, which can be reflected in the splitting of one resonant mode into two resonances in the transmission spectra of the resonator. Thus, the detection can be realized through measuring the resonant frequency splitting (shown in Figure 3).

(a)

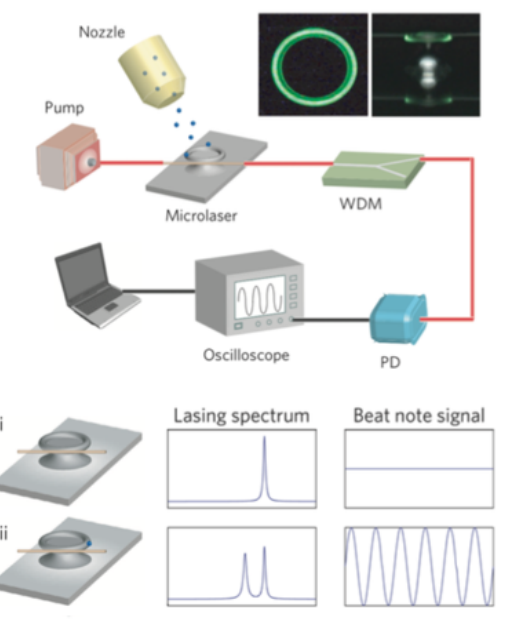

(b)

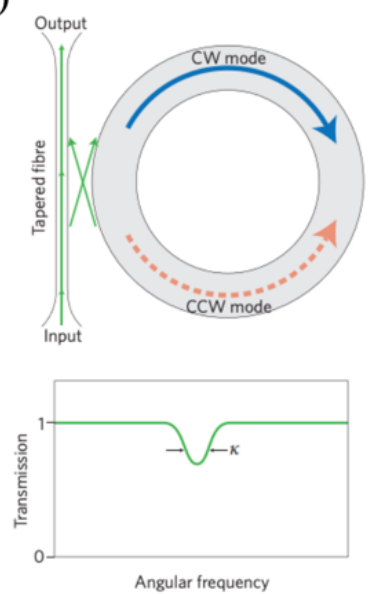

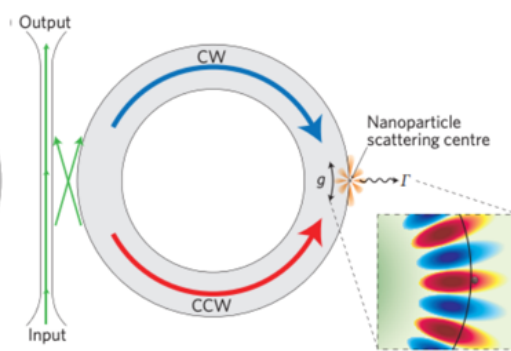

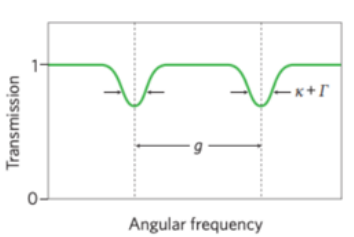

Figure 3. Mode-splitting sensing (a) Schematic of the experimental setup and (i) The lasing spectrum and the beat note signal before nanoparticle binding. (ii) The lasing spectrum and the beat note signal after nanoparticle binding. Reproduced from ref. ${ }^{[40]}$. Copyright 2011 Springer Nature. (b) Field distributions and transmission spectrum of mode splitting in a whispering gallery mode resonator. Reproduced from ref. ${ }^{[54]}$. Copyright 2010 Springer Nature.

This mechanism is mostly exploited for sensing nanoparticles with size down to $10-100 \mathrm{~nm}$ ${ }^{[56,57]}$. It has the advantage of providing a self-referenced sensing since the two split modes share the same noise sources in the surrounding environment ${ }^{[58]}$. In another word, this sensing technique is not influenced by the ambient noises. What's more, it enables the detection of 


\section{WILEY-VCH}

particle size through the observation on the transmission spectra of the resonator ${ }^{[59]}$. One disadvantage of the mode-splitting based sensing is that the $\mathrm{Q}$ factor of the resonator needs to be very high to properly distinguish the mode splitting.

\subsection{Sensitivity enhancement}

Various techniques have been developed for enhancing the detection sensitivity for WGM sensors. In recent years, hybrid plasmon-photonic WGM structures which combine the localization surface plasmon resonances (LSPR) with the WGM resonators have been proposed to effectively enhance the sensitivity ${ }^{[41]}$. The combination is usually realized through coating the resonator with a thin layer of metal ${ }^{[60-62]}$ or attaching plasmonic nanoparticles to the resonator $^{[53,63-65]}$. Taking advantages of both the ultra-low loss characteristics of WGMs and the strong field localization of LSPR modes, these structures normally contain high Q-factor as well as small modal volumes. Plasmonic-enhanced hybrid microresonators have proved their capabilities of lowering the detection limit to single-molecule detection level ${ }^{[26,27,51]}$. B.Q. Shen et al proposed a dissipative sensing method to detect single gold nanorod with a high-Q microtoroid resonator ${ }^{[53]}$. By setting the probe wavelength on and off the surface plasmon resonance, the linewidth change and the mode-shift can be detected simultaneously to evaluate both the reactive and dissipative sensing methods, thus providing more information of the nanoparticles such as the particle polarizability. More recently, the hybrid WGM resonator was further combined with a graphene layer to improve the Raman signals through charge transfer between the graphene and the analyte molecules ${ }^{[66]}$. This hybrid WGM $\mathrm{ZnO} /$ graphene/Ag structure contained three kinds of enhancement factors, namely the strong light-matter interactions provided by WGM resonators, field enhancement by SPR and chemical enhancement by graphene. Thus, a femtomolar surface-enhanced Raman spectroscopy (SERS) 


\section{WILEY-VCH}

response with an enhancement factor up to $10^{12}$ and an ultralow detection limit down to $10^{15} \mathrm{M}$ for probe molecule detection can be realized.

Other approaches including mode locking, active microlasers, exceptional point and ring-up Spectroscopy, have also been proposed to effectively enhance the sensitivity ${ }^{[30]}$. Some of these methods focus on the enhancement based on specific sensing mechanisms. For example, mode locking method ${ }^{[26,67,68]}$ is used to improve time resolution based on mode-shift sensing. Here, the probe laser frequency is locked to the WGM resonance by an electronic feedback loop. The mode-shift signal induced by particle/molecule binding can then be extracted from the direct measurement of the feedback error signal ${ }^{[68]}$. Active microlasers ${ }^{[56,57,69]}$ and exceptional point ${ }^{[70-72]}$ mainly enhance the mode-splitting sensitivity. Compared to passive microresonators which require external laser sources, the microlaser based active WGM resonators can be an excellent alternative platform to release the dependence on the tunable laser source and decrease the costs and bulks of sensing systems. The detection limit is significantly lowered by optical gain, which can be provided through rare earth ion doping (such as erbium, yttrium, thulium doping) ${ }^{[40,73]}$ or stimulated Raman scattering ${ }^{[56,74]}$. In microlaser-based detection, the detection limit is determined by the laser linewidth, which can be made much narrower than the resonance linewidth of any passive resonators ${ }^{[40]}$. This provides opportunities for monitoring small particles/biosamples that are unable to be detected by passive resonators. Through monitoring the beat note signal in the laser output, individual nanoparticles can be continuously detected, including 15-nm-radius polystyrene particles, 10-nm-radius gold particles and influenza A virions ${ }^{[40]}$. Recently, K. Wang et al. developed a novel mechanism to detect nanoparticles with perovskite-based active microresonators ${ }^{[75]}$. They have experimentally demonstrated that the intensity of the scattered laser light shows a monotonously increase with the radius of nanoparticles. In this way, by directly measuring the intensity of laser emission, the detection and sizing of nanoparticles can be achieved with high sensitivity 


\section{WILEY-VCH}

${ }^{[75,76]}$. Unlike the above mentioned techniques, cavity ring-up spectroscopy (CRUS) is able to detect the mode shift, broadening and splitting signals synchronously ${ }^{[77-79]}$. CRUS is an ultrafast approach to acquire spectral snapshots of WGM resonators at minimal time period. By retrieving the ring-up signal and capturing spectra of WGM resonators at time intervals as short as $16 \mathrm{~ns}^{[77]}$, kinetics and events in the nanosecond regime can be monitored.

\section{Integrated sensors based on microfluidic WGM resonators}

The majority of WGM sensors have been realized using bulky and sophisticated laboratory setup under strict environment. Although high sensitivity can be achieved, the WGM resonators need to be carefully aligned with high precision and in a bulky scale ${ }^{[28]}$. Also, the system is very vulnerable and easily affected by the surrounding environment noises. Therefore, there is increasing need in developing a robust and integrated system for WGM resonators. In past few decades, the rapid development of lab-on-a-chip microfluidic systems have opened many new possibilities and provided a solution to this problem ${ }^{[80]}$. The lab-on-a-chip system integrates laboratory work into a chip platform, which aims to increase the robustness and consistency of sensing results and realize the automation of various bioassays. It also offers a way to manipulate sample solutions and implement bioassays with decreased reagent use and reaction time. Combining with the capabilities of optical platform in transducing the biological or chemical signal of the analytes to a quantifiable optical signal with high precision, the concept of photonic lab-on-chip devices have aroused great interest in recent years ${ }^{[81]}$. Therefore, more researches have emerged concerning integrating WGM sensors with microfluidics to build portable lab-on-a-chip devices.

In this section, we roughly divide those WGM resonators into three categories: solid, liquid and hollow resonators. To make it clearer to the readers, we list the advantages and current challenges of different kinds of WGM resonators in Table 1. Then, we focus on the recent 
development of integrating different kinds of WGM sensors with microfluidics, including some packaging methods and integration techniques.

\begin{tabular}{|c|c|c|c|}
\hline \multicolumn{2}{|c|}{$\begin{array}{l}\text { Types of WGM } \\
\text { resonators }\end{array}$} & Advantages & Challenges \\
\hline \multirow{2}{*}{$\begin{array}{l}\text { Solid } \\
\text { resonators } \\
{[29,82]}\end{array}$} & Planar & $\begin{array}{l}\text { Easily fabricated; } \\
\text { Easily integrated into chip scale. }\end{array}$ & $\begin{array}{l}\text { Low detection } \\
\text { sensitivity. }\end{array}$ \\
\hline & Pillar & $\begin{array}{l}\text { Higher detection sensitivity } \\
\text { compared to planar resonators. }\end{array}$ & $\begin{array}{l}\text { Complicated } \\
\text { fabrication procedures; } \\
\text { Mechanical instability. }\end{array}$ \\
\hline \multicolumn{2}{|c|}{$\begin{array}{l}\text { Liquid resonators } \\
\qquad[83,84]\end{array}$} & $\begin{array}{l}\text { Serve as both samples and sensors; } \\
\text { High detection sensitivity; } \\
\text { Tunable sizes and shapes; } \\
\text { Enable real-time sensing. }\end{array}$ & $\begin{array}{l}\text { Liquid evaporation; } \\
\text { Structural instability. }\end{array}$ \\
\hline \multicolumn{2}{|c|}{$\begin{array}{l}\text { Hollow resonators } \\
\qquad[23,85]\end{array}$} & $\begin{array}{l}\text { Structural stability; } \\
\text { High detection sensitivity. }\end{array}$ & $\begin{array}{l}\text { Complicated } \\
\text { fabrication procedures. }\end{array}$ \\
\hline
\end{tabular}

Table 1. Comparison of different types of WGM resonators

\subsection{Solid resonators}

It is known that a vast majority of previous WGM sensors have utilized solid cavities as the resonators. Over these years, considerable efforts have been paid to on-chip WGM sensors since they present great opportunities for integration with microfluidic devices. Many cavity 


\section{WILEY-VCH}

geometries have been proposed, which can roughly be divided into two categories: planar and pillar supported resonators ${ }^{[29]}$.

Micro-rings ${ }^{[86-92]}$ and micro-disks ${ }^{[93,94]}$ are typical planar cavities. Many on-chip WGM sensors based on planar resonators have been proposed recently, which enables label-free, realtime sensing and detection in an integrated platform ${ }^{[95-99]}$. In particular, silicon-based resonators have been widely studied owing to their advantages in photonic integration. R. C. Bailey's group has developed a sensing platform based on silicon microring resonator arrays ${ }^{[100]}$. Sensor chip with a dimension of $6 \times 6 \mathrm{~mm}$ which consists of 64 microring resonators (30 $\mu \mathrm{m}$ in diameter) was fabricated on silicon-on-insulator wafers. Each of the microring was addressed by a linear waveguide, which allowed the independent recording of the optical spectrum for individual microring resonator. This platform has been experimentally demonstrated to be useful in many application scenarios including multiplexed biosensing ${ }^{[101]}$ and mass concentration detection ${ }^{[102]}$. Q. Song's group has developed a novel technique for mass fabrication of high-Q silicon microdisks ${ }^{[103]}$. The limitation of standard photolithography method including high surface roughness and low Q-factor can be overcome through utilizing isotropic etching process. This technique can effectively smooth the surface of silicon microdisks to a roughness less than $15 \mathrm{~nm}$. In another work, they have also experimentally demonstrated a cost-effective and simple mechanism to couple light into the high-Q silicon microdisk. They connected a waveguide to the silicon microdisks so that the incident light can be injected into the resonator directly through the waveguide without using the evanescent coupling setting ${ }^{[94]}$. This end-fire injection configuration can achieve a coupling efficiency up to $57 \%$ and a Q-factor at the level of $10^{5}$. These researches have paved the way for mass fabrication as well as practical applications of silicon-based resonators. Figure 4 (a)(b) shows one example of integrated micro-ring WGM sensor ${ }^{[104]}$. In this design, the polymer WGM microresonator sensor was integrated inside a glass microfluidic chip. The device fabrication 


\section{WILEY-VCH}

process includes microfluidic chip fabrication through hybrid femtosecond laser micromachining, freestanding polymer sensor fabrication by two photon polymerization (2PP) method and assembling with PEEK tubes and optical fibers. This integrated WGM sensor has been exploited for label-free biosensing with a high refractive index sensitivity of up to $61 \mathrm{~nm}$ per RIU. Furthermore, in the biotin-streptavidin binding experiment, the minimum detectable surface density increase can reach $67 \pm 5 \times 10^{3}$ molecules per $\mu \mathrm{m}$. In Figure 4 (c), a micro-ring resonator integrated with poly-(dimethylsiloxane) microfluidic channels was illustrated by S. $\mathrm{Wu}$ et al ${ }^{[105]}$. The incorporation of microfluidic channels not only significantly decreased the sample consumption and experimental cost, but also made the modification of the chip more efficient. The real-time and quantitative detection of $\mathrm{IgG}$ with a detection limit of $0.5 \mu \mathrm{g} / \mathrm{ml}$ was demonstrated, which is 14 times lower than previously reported microring resonators.

Generally, planar resonators are limited to a low detection sensitivity due to the low Q factor caused by their direct contact with the substrate. As a result, several methods were proposed to increase the sensitivity, including employing slot waveguides ${ }^{[106]}$, using porous silicon resonators ${ }^{[107]}$, cascading the resonators ${ }^{[108-110]}$ and amplifying signals through particle trapping ${ }^{[111]}$, etc. 


\section{WILEY-VCH}

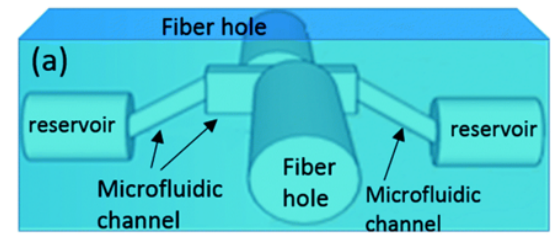

(c)
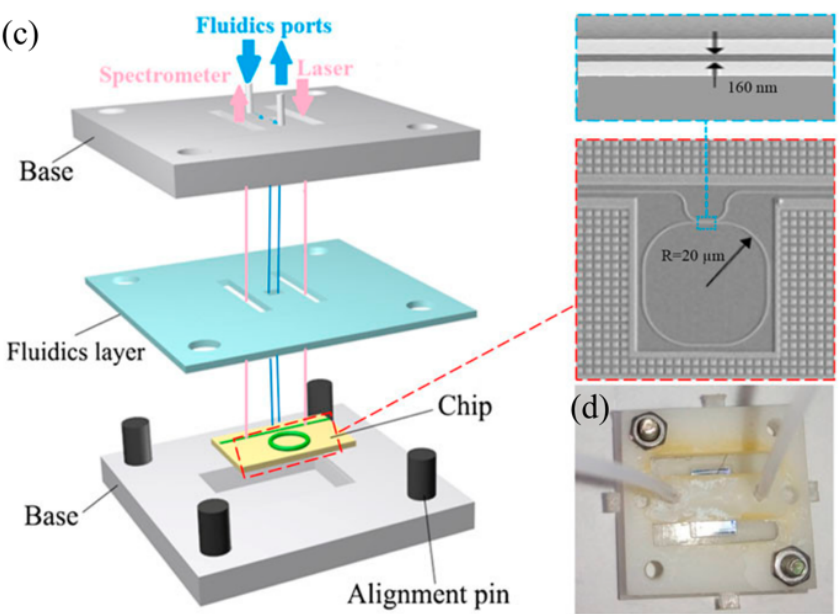

Figure 4. On-chip planar resonators for sensing. (a) Schematic view of the glass microfluidic chip; (b) detail of the WGM biosensor integrated inside the microfluidic channel. Reproduced from ref. ${ }^{[104]}$ with permission of Royal Society of Chemistry. (c) Schematic illustration of the integrated sensing system. (d) Photograph of the PDMS microfluidic channels. Reproduced from ref. ${ }^{[105]}$ with permission of AIP Publishing.

To overcome the challenge of low sensitivity, pillar supported resonators, namely microspheres ${ }^{[27,112,113]}$, microgoblets ${ }^{[114,115]}$ and microtoroids ${ }^{[38,116]}$, which can lead to a much higher detection sensitivity due to their high Q factor compared to planar resonators, have been exploited. Nonetheless, the fabrication process is more complicated. The requirement of tapered fiber as the coupling device also results in mechanical instability. Thus, many studies have been carried out to fabricate chip-scale pillar supported resonators. Although the incorporation of silicon substrate facilitates photonic integration ${ }^{[117]}$, it still has several disadvantages including its biocompatibility difficulties and strong absorption of visible light, which hinders the sensing

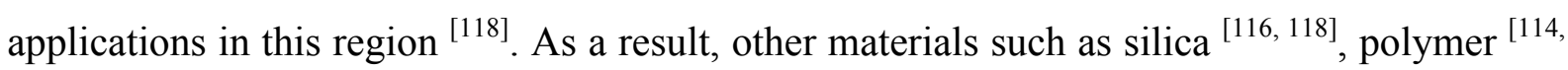
${ }^{119]}$, silicon nitride ${ }^{[120]}$ and chalcogenides ${ }^{[113]}$, have been explored to fabricate microresonators. For example, Figure 5 (a) shows the fabrication process of all-glass microtoroid resonators ${ }^{[18]}$. The fabrication process preserves the same scheme as $\mathrm{SiO}_{2}$-on-Si microtoroids but removes the 


\section{WILEY-VCH}

silicon pillar and results in a silicon toroid atop an oxide pillar. The elimination of silicon largely facilitates large-scale integration and enables fluorescence detection. The integration of microtoroid resonator with waveguide was also reported by X. Zhang et al ${ }^{[121]}$. Through the combination of top down and bottom-up fabrication methods, they packaged silica microtoroid with an on-chip waveguide to form a monolithically integrated microtoroid-waveguide system. This system offers the advantages of high quality factor which exceeds 4 million and wavelength flexibility, which is of great importance in sensing applications. T. Wienhold et al ${ }^{[114]}$ proposed an all-polymer sensing platform based on microgoblet resonators (Figure 5 (b)). The microgoblet resonator was fabricated through spin-coating, optical lithography, wet chemical etching, and parallel thermal reflow procedures. Further, they exploited this platform to perform refractive index sensing, which shows a high sensitivity of $10.56 \mathrm{~nm}$ per refractive index unit. This integrated microfluidic chip based on all-polymer WGM resonators has the advantages of low fabrication cost and high throughput, thus has great potential in promoting disposable chips with low cost and high sensitivity in sensing platform for point-of-care diagnostics.

(a)

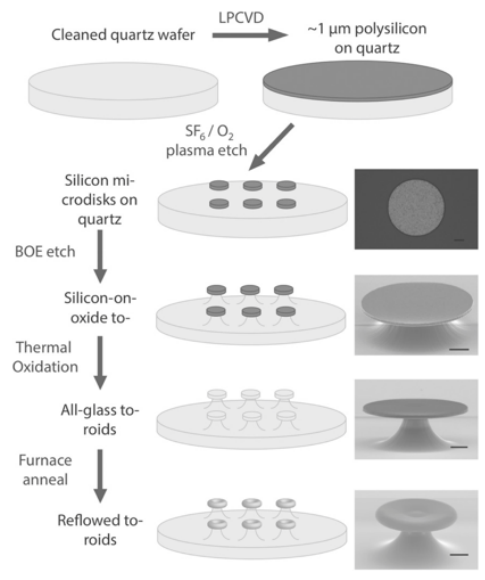

(b)

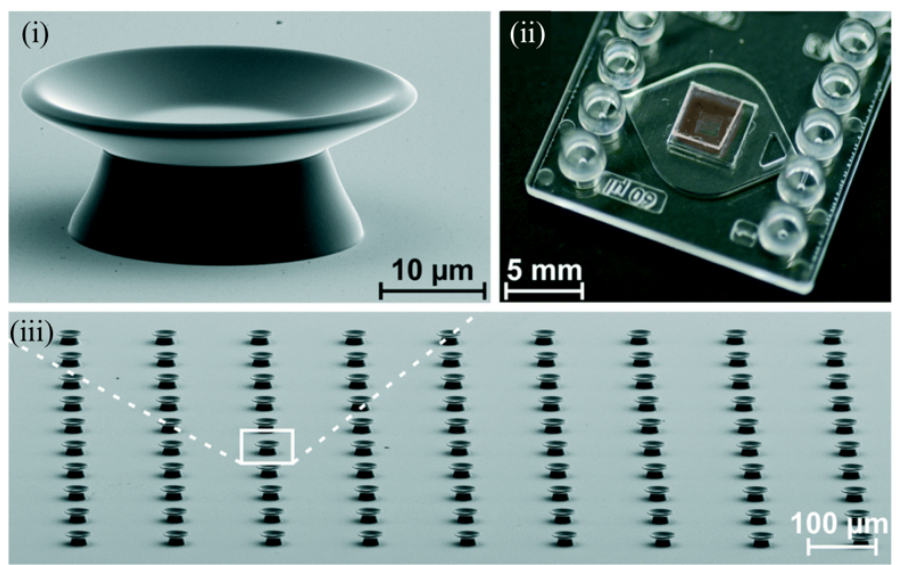

Figure 5. (a) Fabrication process of all-glass toroid resonators. Reproduced from ref. ${ }^{[118]}$ with permission of John Wiley and Sons. (b) PMMA microgoblet lasers fabricated on a polysulfone substrate: (i) Zoom-in of a single PMMA microgoblet laser fabricated on a polysulfone substrate. (ii) Array of 100 microgoblet 


\section{WILEY-VCH}

lasers fabricated by parallel solution-based processing. (iii) Photograph of a sensing chip. Reproduced from ref. ${ }^{[114]}$ with permission of Royal Society of Chemistry.

To date, most studies on WGM resonators have utilized a tapered fiber to efficiently couple light into the resonator. However, this coupling system are limited by environmental disturbance because of its low mechanical stability, which will affect the sensing precision and hinder their wider applications. To overcome this challenge, several researchers have designed the packaging strategies for WGM sensors to form an integral structure. These packaged systems are not only more reliable due to the increase in stability of the coupling between the fiber and WGM resonator, but also more portable and integrated. Here, two main strategies are most commonly presented: (i) wholly-package; and (ii) spot-package ${ }^{[1]}$. Wholly-package is a technique of enclosing the entire coupling system with a low refractive index (RI) material. Y.Z. Yan et al ${ }^{[122]}$ proposed a packaged microsphere-taper coupling structure using the low RI ultraviolet glue as the coating material. This method has successfully isolated the microspheretaper from the surrounding environment and managed to maintain the Q-factor above $10^{6}$ for a few months. F. Monif et al ${ }^{[123]}$ encapsulated a microtoroid resonator with a coupled fiber taper using low RI polymer. This system was tested under various ambient conditions to have high robustness with a high sensitivity of $0.131 \mathrm{~nm} /{ }^{\circ} \mathrm{C}$ for thermal sensing, which is 10 times higher than unpackaged silica microtoroid. Wholly-packaged design provides protection for the WGM resonator from the external perturbance. Though being able to isolate the environmental noise, this scheme also isolates analytes at the same time, which makes it unable to realize biochemical sensing through measuring interactions of the evanescent field with analytes. Moreover, the coupling conditions will be affected in the package process and Q factor will be decreased due to the optical loss of the UV polymer. As a result, some researchers designed the spot-package method for packaging WGM resonators. In this strategy, only the coupling region was encapsulated with low RI polymer ${ }^{[124]}$. Compared to wholly-package method, this structure 


\section{WILEY-VCH}

is able to maintain the ability of interacting with the analytes. However, operation of this packaging technique is relatively difficult. Also, due to the fact that smaller package spot will decrease the system robustness while larger point will reduce the Q factor, the size of package spot should be carefully controlled to reach a balance between good stability and high Q-factor. There are also some other approaches to address this issue. Some special microresonators were also designed and optimized to allow stable and controllable coupling by placing the fiber taper in contact with the resonator. For instance, inverted-wedge resonators were designed so that the thin outer ring of the resonator can provide support for the coupling fiber, which leads to robust coupling status ${ }^{[125]}$. Moreover, an octagonal toroidal microcavity were fabricated to enhance the mechanical stability of coupling by allowing the tapered fiber to touch the sidewall of the cavity ${ }^{[126]}$. Furthermore, coupling systems in the form of in-fiber couplers possessing the advantages of compactness and robustness have also been reported ${ }^{[127-129]}$.

There have been many efforts to exploit the integration of WGM sensors with microfluidics. These microfluidic integrated WGM platforms provide promising opportunities in real-time biodetection and point-of-care diagnostics. X. Xu et al ${ }^{[130]}$ developed a compact WGM system with multiple components including a tunable laser, a function generator and a photodiode detector and demonstrated its application in temperature sensing. This system, though have not been fully integrated with microfluidics, provides some insight in terms of combing and

integrating different components for LOC devices. A. I. Gubin et al ${ }^{[131]}$ reported a technique based on a WGM resonator covered by a plastic layer with a microfluidic channel to measure the permittivity of liquids. The liquids were measured inside a microfluidic channel on a top of the WGM resonator, which not only ensures the contactless measurement but also increases the sensitivity. Through analyzing the interaction of electromagnetic field with liquid in a WGM resonator and measuring both the WGM resonance frequency shift and the change of the inverse quality factor, the small concentration change in biochemical solutions can be detected with 


\section{WILEY-VCH}

high accuracy. This approach was applied to obtain the complex permittivity for small volume of sample solutions such as glucose, albumin bovine serum, lactalbumin and cytochrome C.

Apart from the integration with channel-based microfluidics with relatively bulk liquid volume, some work has also been explored in using droplet microfluidic to further decrease the reagent volume and reaction time. For example, S.M. Wildgen et al ${ }^{[132]}$ proposed to use WGM resonators in small droplets to facilitate rapid analyte detection. They loaded 500 bariumtitanate microsphere resonators into a $10 \mu \mathrm{L}$ PBS droplet on a hydrophobic surface. WGM resonances were excited by the evanescent field at the substrate interface, which is very sensitive to the refractive index change caused by the binding of biomolecules onto the functionalized WGM resonators. Each resonator can serve as an individual assay, which enables multiplexed sensing with extremely small volumes. This study is very useful in rapid biosensing applications where fast mass transfer of the analyte and quick response time upon analyte binding are required. L. Luan et al integrated a microdisk resonator and a photodiode into a digital microfluidic (DMF) system ${ }^{[133]}$. In DMF systems, each droplet serves as an isolated microreactor, which is easily manipulated and convenient to conduct parallel experiments. The integration allows for sample preparation of different concentrations in droplets and refractive index change detection through measuring the electrical current signal transduced from the optical output. Though multiplexed sensing was not supported in this work, it is a significant step towards the integration of optical systems, which has great potential in clinical diagnostics. More recently, S.F. Wondimu et al have successfully integrated multiple whispering-gallery mode sensors into the DMF system for multiplexed sensing ${ }^{[134]}$. In this setup, microgoblet resonators are integrated on the top substrate of the DMF assembly. The WGM resonators were doped with organic dyes so that they can function as optically pumped lasers. Excitation and read-out of the microgoblet lasers can be realized via free-space optics, which enables largescale integration. On the bottom substrate of the system, actuation electrodes are placed to 


\section{WILEY-VCH}

manipulate droplets (shown in Figure 6). Both refractive index sensing and label-free detection were demonstrated using this system. The experimental results show a refractive index

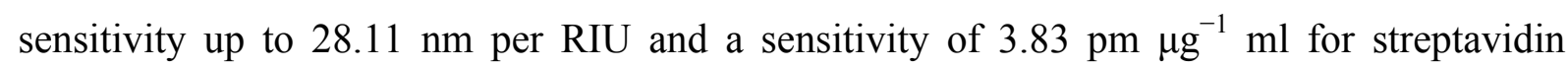
detection. Combining the advantages of both droplet microfluidics and WGM resonators, this technique enables in situ label-free detection of multiple analytes in a single DMF cell.

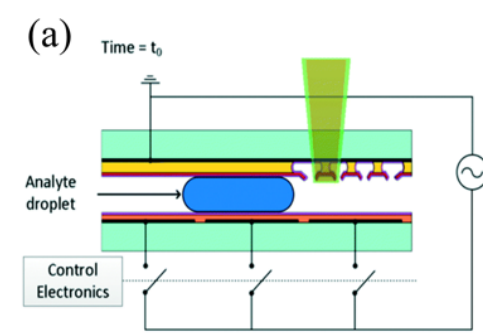

(b)

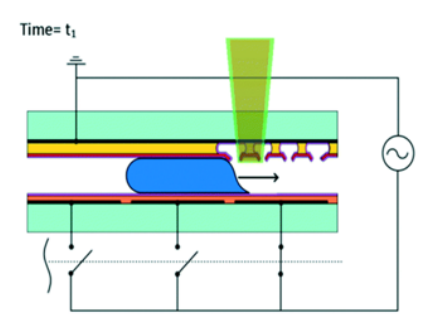

(c)
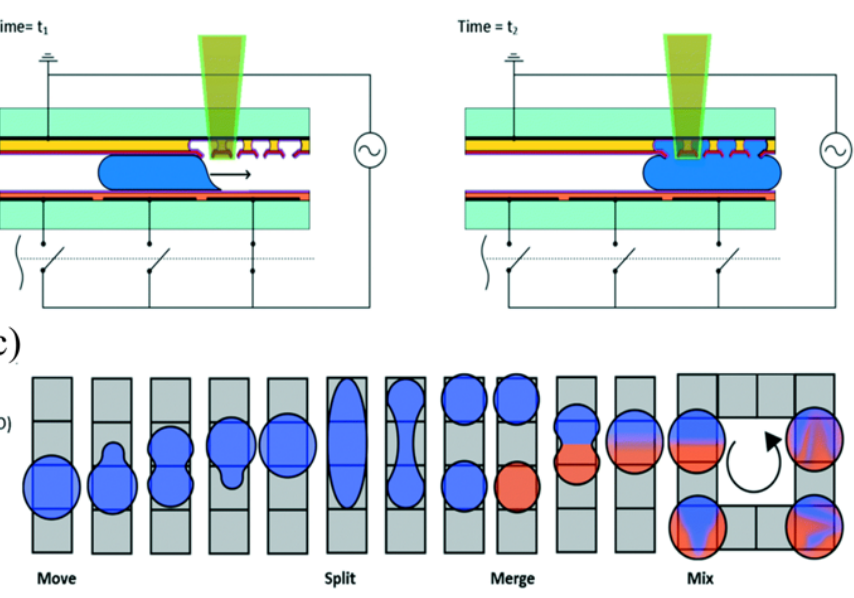

Figure 6. Cross-sectional view of a digital microfluidic system integrated with microgoblet resonators. (a) Analyte delivery process through activating the electrodes. (b) Close-up of the integrated system depicting the material layers (c) Some basic on-chip microfluidic operations. Reproduced from ref. ${ }^{[134]}$ with permission of Royal Society of Chemistry.

\subsection{Liquid resonators}

Although solid resonators have been widely adopted in WGM sensors, they have some fundamental disadvantages. One important issue is that the shift in WGM resonance frequency is small since samples only interact with the relatively weak evanescent tail in external medium. Also, long detection time is often required due to the delivery of samples and the requirement of reaching surface equilibrium conditions.

As a result, some researchers have proposed to use micro-resonators which are directly made of liquid droplets containing the reagents to be detected. In this way, droplets serve as 


\section{WILEY-VCH}

both the samples and the sensors. Liquid resonators were studied since $1970 \mathrm{~s}^{[135]}$. Over these years, many new designs of liquid resonators have been proposed. Typically, a capillary ${ }^{[136]}$ or cylindrical stem ${ }^{[137]}$ is used to sustain a droplet as the resonator. When a laser beam is close to the droplet rim, the WGM spectrum of the liquid resonator is formed. This setting ensures that target molecules are able to interact with the stronger portion of the WGM, which fully exploited the cavity enhancement of WGM, thus leading to much higher detection sensitivity ${ }^{[84]}$. Another key advantage of liquid resonators is their tunable sizes and shapes $[138,139]$ compared to solid resonators which have fixed geometries after fabrication. Liquid resonators also allow for frequency-tunable resonance modes with external stimulus such as electrical control ${ }^{[140]}$ or surface tension alteration ${ }^{[141]}$. Furthermore, utilizing liquid resonators enables real-time sensing and detection of the samples.

Liquid resonators are suitable for integration with microfluidic manipulation or analysis platforms owing to their low-cost and convenient fabrication process. The integration has many notable advantages. Firstly, it is obvious that the microfluidic chip offers a sealed place for bioreactions, which helps reduce the perturbations from the environment. Furthermore, the microfluidic channel provides an effective way to generate and manipulate droplets. Through microfluidic channel design, one can control the size of droplets to realize the tuning of WGM resonance $^{[138]}$. There is also report in utilizing silicone rubber to hold and manipulate droplets ${ }^{[142]}$. A droplet array can be designed through this method, which holds great potential in many novel applications due to the combination of the intra- and inter-cavity resonances. Moreover, the integration with microfluidic chips provide a convenient way to introduce many other unit functions into the system. For example, one can embed heating modules inside a microfluidic chip to control the temperature of liquid resonator, which realizes real-time thermal tuning ${ }^{[143]}$. To facilitate effective integration, many free-space coupling techniques have been exploited ${ }^{[144-147]}$. For example, some researchers inserted optical fibers into the chips and trap the droplet 


\section{WILEY-VCH}

inside the microfluidic channel with optical tweezers ${ }^{[145]}$, which provides a feasible way to trap droplet resonators inside microfluidic channels and perform sensing experiments. As shown in Figure 7 (a), droplets with different size can be trapped by optical tweezers near the tip of an excitation fiber such that WGMs inside droplets can be excited with a tunable laser. Q-factors of WGMs up to $1.8 \times 10^{4}$ were measured for droplets with a diameter of $45 \mu \mathrm{m}$. Spectral shifts of WGM were also observed due to droplet dissolution, which shows its capability of sensing minute perturbations. Complex systems such as double emulsions can also be realized through the integration of microfluidic chips. L. Zheng et al ${ }^{[148]}$ designed a microfluidic system with embedded liquid lasers. Double emulsion droplets were generated through two microfluidic capillary devices in series (Figure 7 (b)). In particular, they designed two modes of employing laser droplets: the benzyl alcohol droplets contained dye solutions were either embedded within or wrapped around aqueous microfluidic droplets. Both cases were characterized in terms of lasing behavior through detecting WGM resonances. The experimental results show great potential in realizing on-drop sensing through WGM-based lasing. L. J. Chen et al ${ }^{[149]}$ also proposed a double emulsion system facilitated by microfluidics, where the droplet's position can be controlled using a magnet. Although further sensing experiments are not performed in this article, the idea of employing liquid crystals droplets as WGM microresonators in a microfluidic context is inspiring. The high Q-factor as well as the electric-field tunability of WGM lasers presented in this system have great potential in various sensing applications. 


\section{WILEY-VCH}

(a)

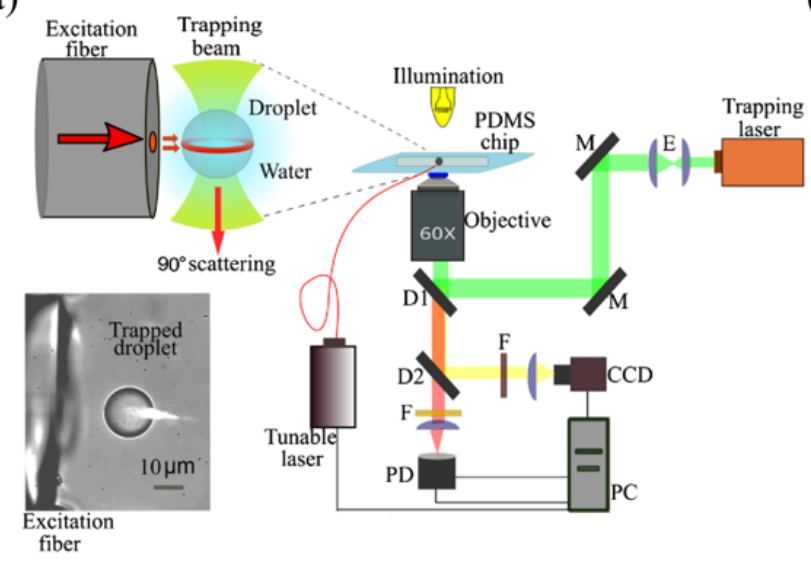

(b)

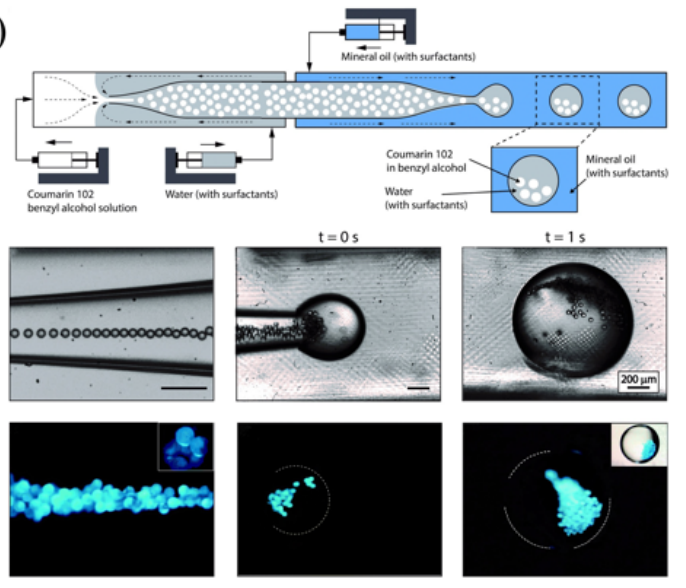

Figure 7. (a) Experimental setup of microdroplet scattering experiments and an image of scattered light when the trapped droplet was excited by a red laser. Reproduced from ref. ${ }^{[145]}$ with permission of The Optical Society. (b) Glass capillary devices to embed liquid lasers within aqueous microfluidic droplets. Reproduced from ref. ${ }^{[148]}$ with permission of Wiley Online Library.

\subsection{Hollow resonators}

Despite the advantages provided by liquid resonators, they have the drawbacks of liquid evaporation and structural instability, which greatly limits their practical applications. Therefore, many researchers have proposed to solidify the media surrounding liquid droplets. This type of resonators with thin spherical shells and hollow volume inside can be summarized as hollow resonators ${ }^{[85]}$. In this way, the resonators not only have increased stability compared to liquid resonators, but also maintain most advantages of liquid resonators compared to solid ones such as high sensitivity and convenient integration with microfluidics. Hollow resonators can be roughly divided into two categories: bottle-like resonators ${ }^{[150-152]}$ and the microbubble resonators ${ }^{[153,154]}$. The latter is more commonly adopted owing to its high Q-factor. They are usually fabricated through local heating on a silica microcapillary while pressurizing the cavity internally. The local heating and internal pressure will determine the cavity shape and shell thickness. For example, a single-mode fiber embedded microbubble resonator with compact size and low cost as a highly sensitive pressure sensor was reported ${ }^{[155]}$. The microbubble 


\section{WILEY-VCH}

resonator with wall thickness down to $2 \mu \mathrm{m}$ was fabricated by utilizing the pressure-assisted arc discharge technique. This ultrathin resonator can provide a pressure sensitivity of 164.56 $\mathrm{pm} / \mathrm{kPa}$ and a temperature sensitivity of $4 \mathrm{pm} /{ }^{\circ} \mathrm{C}$.

Recently, researchers have focused on tuning the WGM resonance through temperature ${ }^{[156]}$, strain $^{[157-159]}$ or pressure ${ }^{[160-162]}$, which has laid a foundation for different sensing applications using microbubble resonators. Some researchers also studied the optimized geometrical parameters of microbubble resonators for sensing ${ }^{[163]}$. Other than theoretical calculations and simulations, more and more researchers have focused on constructing integrated microbubble WGM sensors for sensing applications. In the most commonly used setup, the sample fluid ${ }^{[164 \text {, }}$ ${ }^{165]}$ or air ${ }^{[23]}$ is passing through the microbubble resonator for detection, usually by connecting to a syringe pump or a compressed air source while a tunable laser source is coupled into the hollow WGM resonator through a tapered optical fiber.

Like solid resonators, many packaging strategies have also been proposed for hollow resonators. T. Tang et al showed an example of packaging the microbubble resonator with the fiber ${ }^{[166]}$. Figure 8 (A) shows the four steps of packaging process, including optimal coupling of the microresonator and the fiber taper, fixation of the resonator, injection and solidification of UV polymer. The packaged system is capable of conducting sensing experiments in aqueous environment. The sensing performance were evaluated through refractive index sensing and surface sensing, which shows a sensitivity of $18.8 \mathrm{~nm} / \mathrm{RIU}$ and $31.29 \mathrm{pm} / \mathrm{nm}$. Besides microbubble resonators, there has also been progress in the fabrication and packaging based on microbottle resonators. G. Persichetti et al ${ }^{[167]}$ proposed a self-assembled and packaged microbottle resonators in a full polymeric lab-on-a-chip sensing platform. In this packaging scheme (Figure 8 (B)), a planar SU-8 waveguide was fabricated instead of the commonly used tapered fiber to enhance the stability and a fiber stem was used to mechanically support the microcavity without affecting its optical properties. Two holes are drilled at the ends of fiber 


\section{WILEY-VCH}

stem, which allows for the controllable delivery of glue. This spot-package technique effectively prevents packaging directly on the coupling region and is able to perform biochemical sensing. This platform has a refractive index sensitivity of $120 \mathrm{~nm} / \mathrm{RIU}$. Possessing the advantages of high sensitivity, good portability and robustness, packaged hollow WGM sensors can be exploited in integrated photonic devices for various sensing applications.

(A)

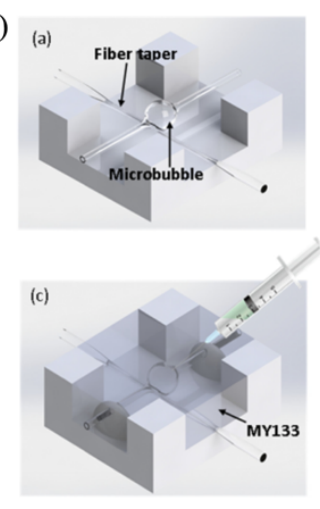

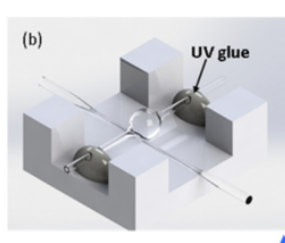

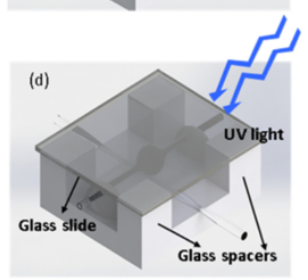

(B)

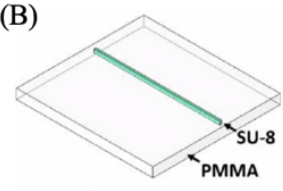

(a)

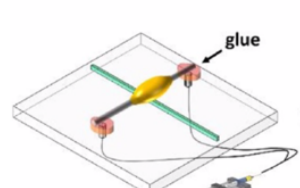

(d)

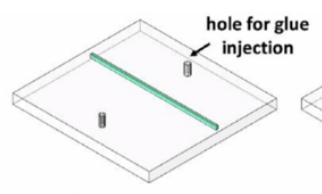

(b)

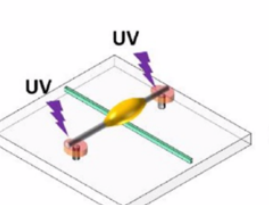

(e)

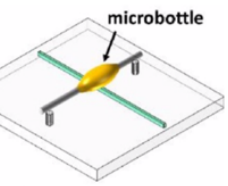

(c)

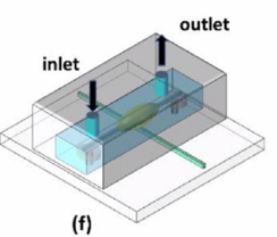

Figure 8. (A) Packaging process of microbubble resonators. Reproduced from ref. ${ }^{[166]}$ with permission of Optical Society of America. (B) Lab-on-chip platform fabrication and packaging steps of microbottle resonators. Reproduced from ref. ${ }^{[167]}$ with permission of Elsevier.

\section{Applications}

WGM resonators have been exploited in a wide range of sensing applications due to the strong dependence of the mode changes on the resonator geometry and surrounding environment ${ }^{[168]}$. Here we briefly review some of the latest development in different sensing applications, including nanoparticle and biomolecule sensing, temperature, humidity and pressure sensing.

\subsection{Nanoparticle and biomolecule sensing}




\section{WILEY-VCH}

WGM sensors have been widely used in sensing applications because of their capability of detecting particles or biomolecules in a label-free manner. Here we briefly review some of the latest progress in these areas.

Nanoparticle sensing has drawn significant attention from the research field during the past few years. Various geometries of resonators and sensing mechanisms have been exploited to improve the sensitivity. For example, using liquid resonators instead of solid resonators can greatly enhance the detection sensitivity ${ }^{[84]}$. Microbubbles have been proved to have much better performance than microsphere resonators in particle sensing ${ }^{[163]}$. J. M. Ward et al ${ }^{[169]}$ have successfully demonstrated that using microbubble resonators can largely increase the detection sensitivity beyond the limitation of the evanescent field. The experimental results show that in mode-broadening based sensing for $100 \mathrm{~nm}$ particles, there is 276 times increase in terms of the number of linewidths compared with microsphere resonators. More recently, $\mathrm{H}$. Jing et al ${ }^{[170]}$ use a spinning a WGM resonator other than the commonly used stationary one to achieve much higher sensing performance. The mode-splitting based sensing is enhanced due to the different sagnac frequency shifts experienced by the clockwise and counterclockwise optical fields during spinning. In Table 2, we have summarized the performance of different types of the WGM sensors for nanoparticle and biomolecule sensing in recent papers.

\begin{tabular}{|c|c|c|c|c|}
\hline Types & Analytes & Strategy & Performance & Ref \\
\hline \multirow[t]{2}{*}{ Microring } & $\begin{array}{l}\text { Biotin-- } \\
\text { streptavidin }\end{array}$ & $\begin{array}{l}\text { Fabricated by hybrid } \\
\text { femtosecond laser } \\
\text { micromachining }\end{array}$ & $\begin{array}{l}\text { Detection limit: } 0.0048 \pm \\
0.0003 \text { RIU } \\
\text { Sensitivity: } 61 \pm 1 \mathrm{~nm} \text { per } \\
\text { RIU }\end{array}$ & [104] \\
\hline & $\begin{array}{l}\text { Bovine serum } \\
\text { albumin }\end{array}$ & $\begin{array}{l}\text { Ultrahigh Q polymer } \\
\text { microring }\end{array}$ & Detection limit: $5.3 \mathrm{pg} / \mathrm{mm}^{2}$ & [171] \\
\hline Microdisk & $\begin{array}{c}\text { NACL } \\
\text { Protein A }\end{array}$ & $\begin{array}{l}\text { Silicon photonic micro- } \\
\text { disk }\end{array}$ & $\begin{array}{c}\text { Detection limit: } 5.5 \times 10^{-4} \\
\text { RIU }\end{array}$ & [172] \\
\hline
\end{tabular}


WILEY-VCH

\begin{tabular}{|c|c|c|c|c|}
\hline & $\begin{array}{l}\text { Anti-streptavidin } \\
\text { biotin-BSA }\end{array}$ & & Sensitivity: $142 \mathrm{~nm}$ per RIU & \\
\hline & Human IgG & $\begin{array}{c}\text { Active hyperboloid-Drum } \\
\text { Microdisk }\end{array}$ & Detection limit: $9 \mathrm{ag} / \mathrm{ml}$ & [173] \\
\hline & Streptavidin & $\begin{array}{l}\text { Novel referencing scheme } \\
\text { Microdisk laser arrays }\end{array}$ & $\begin{array}{l}\text { Detection limit: } 104 \mathrm{ng} / \mathrm{ml} \\
\text { Sensitivity: } 13 \mu \mathrm{g} / \mathrm{ml}\end{array}$ & [174] \\
\hline \multirow[t]{3}{*}{ Microsphere } & Neutravidin & $\begin{array}{l}\text { Two microspheres in } \\
\text { microstructured optical } \\
\text { fiber }\end{array}$ & Detection limit: $0.20 \mu \mathrm{g} / \mathrm{mL}$ & $\begin{array}{l}{[175,} \\
176]\end{array}$ \\
\hline & B. globigii & $\begin{array}{l}\text { Quantum Dot-coated } \\
\text { Microsphere }\end{array}$ & Precision: $3 \times 10^{-4}$ in $\mathrm{RI}$ & [177] \\
\hline & $\begin{array}{l}\text { Helicobacter } \\
\text { hepaticus }\end{array}$ & Silica microspheres & $\begin{array}{l}\text { Detection limit: } \\
1 \times 10^{4} \text { cells } / \mathrm{mL}\end{array}$ & [178] \\
\hline \multirow[t]{3}{*}{ Microtoroid } & Exosome & $\begin{array}{l}\text { Digital } \\
\text { frequency locking }\end{array}$ & $\begin{array}{l}\text { Accuracy in mode shifts: } 10 \\
\text { attometers } \\
\text { Detection limit: single } \\
\text { exosome with size of } 44 \pm \\
4.8 \mathrm{~nm}\end{array}$ & [24] \\
\hline & $\begin{array}{l}\text { Human } \\
\text { interleukin } 2\end{array}$ & $\begin{array}{l}\text { Robust, silane- based } \\
\text { surface modification }\end{array}$ & Detection limit: $0.1 \mathrm{nM}$ & [179] \\
\hline & $\begin{array}{l}\text { Human chorionic } \\
\text { gonadotropin }\end{array}$ & $\begin{array}{l}\text { Frequency locked } \\
\text { resonators }\end{array}$ & Detection limit: $3 \mathrm{fM}$ & [67] \\
\hline \multirow[t]{3}{*}{ Microbubble } & Silica molecules & $\begin{array}{l}\text { Packaged system using } \\
\text { polymer }\end{array}$ & 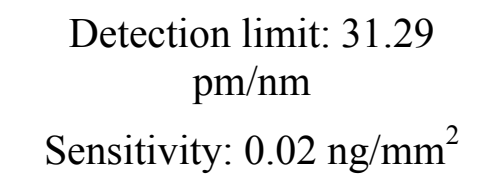 & [166] \\
\hline & $\begin{array}{c}\text { E. coli } \\
\text { paraoxon }\end{array}$ & $\begin{array}{l}\text { PVA-Lysozyme } \\
\text { microbubbles }\end{array}$ & Detection limit: $0.5 \mathrm{ppm}$ & [180] \\
\hline & Lead ions & $\begin{array}{l}\text { GR-5 DNAzyme } \\
\text { modification }\end{array}$ & $\begin{array}{c}\text { Detection limit: } 15 \mathrm{fM} \\
\text { Sensitivity: } 265.2 \mathrm{~nm} \text { per } \\
\text { RIU }\end{array}$ & [181] \\
\hline Microbottle & $\begin{array}{l}\text { Bovine serum } \\
\text { albumin }\end{array}$ & $\begin{array}{c}\text { Self-referenced } \\
\text { differential-mode detection } \\
\text { scheme }\end{array}$ & Detection limit: $10 \mathrm{fg} / \mathrm{ml}$ & [182] \\
\hline
\end{tabular}




\section{WILEY-VCH}

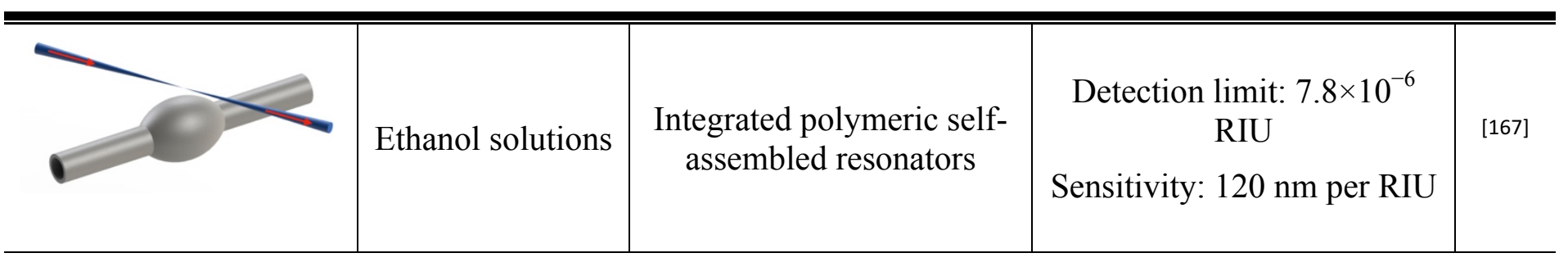

Table 2. Performance of different types of the WGM resonators for biosensing

In the particle sensing process, it is important to deliver target particles precisely to the sensing region. To solve the limitation due to the reliance on random diffusion, $\mathrm{K}$. Han et al ${ }^{[183]}$ use an optomechanical sensor to enable the detection of flowing particles with high throughput. It is also highlighted that the mechanical properties of particles including mass density, viscoelastic dissipation, mechanical compressibility and elastic modulus can be measured. The experimental results indicate that these optical measurements can be realized at rates exceeding 10,000 particles per second. The group also demonstrated real-time sensing with electro-optomechanics ${ }^{[184]}$. The proposed scheme has a temporal resolution of better than $20 \mu$ s and particle size sensing resolution down to $490 \mathrm{~nm}$. Moreover, there is some progress in making on-chip single particle sensing and imaging systems ${ }^{[111,118]}$, which facilitates large-scale integration.

Nanoparticle sensing has opened many possibilities for biological applications and clinical diagnostics, where the presence and characteristics of analyte molecules need to be accurately detected and quantitatively analyzed. To date, many biomolecules have been detected, including viruses ${ }^{[40]}$, bacteria ${ }^{[178,185]}$, DNA ${ }^{[51]}$, RNAs ${ }^{[186]}$, proteins ${ }^{[27,187]}$, exosomes ${ }^{[24]}$ and ribosomes ${ }^{[26]}$. Researchers are now able to detect not only the presence of biomolecules, but also the information regarding the concentration ${ }^{[188]}$, size and mass ${ }^{[38,44]}$ of the analyte through the signal change of WGM sensors. In biosensing applications, it is also of vital importance to monitor the specific interactions between molecules, which has been reported in many cases ${ }^{[25,}$ 51]. 


\section{WILEY-VCH}

(a)

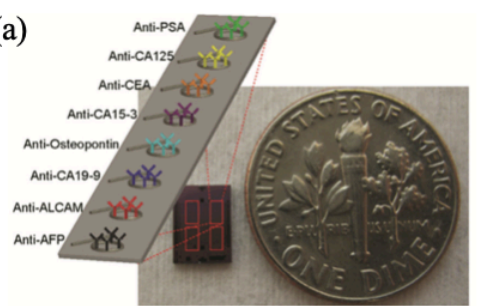

(b)

(c)
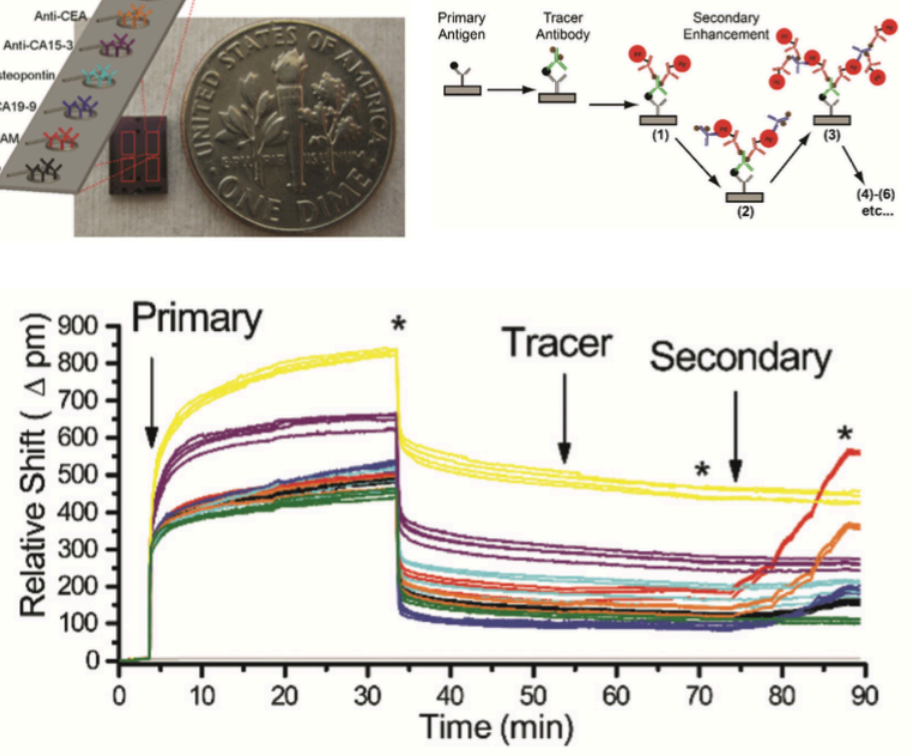

Figure 9. (a) Silicon microchip overlaid with four red boxes indicating sub-array regions containing 8 microring resonators, each functionalized with an antibody specific for a different cancer biomarker antigen. (b) Methodology of the layer-by-layer signal enhancement on the microring resonator silicon surface. (d) A real-time plot showing the resonance wavelength shifts through the entire bioassay. Reproduced from ref. ${ }^{[189]}$ with permission of Royal Society of Chemistry.

It is also worth mentioning that the recent development of integrating microfluidic chips with biosensors has paved the way for multiplexed sensing. Through the proper design of microfluidic systems, large quantities of parallel experiments from sample loading to controlled bio-reactions can be performed ${ }^{[190]}$. Thus, combining with WGM resonators, which can also be cascaded as arrays to realize multiple sensing, an integrated system with parallel processing and multiplexed sensing abilities can be built. To elaborate, an array of microring resonator integrated with microfluidic chips in a compact cartridge was proposed to realize multiplexed biosensing ${ }^{[106]}$. The integrated cartridge includes an optical chip, a microfluidic layer, an adhesive film and a plastic shell. Light is split and coupled to six microring resonators in different channels, which are all individually addressable by a microfluidic channel containing input and output ports to transport different analytes. This work shows the capability of using 


\section{WILEY-VCH}

integrated WGM sensor array to perform multiple sensing experiments in parallel. A.L. Washburn et al ${ }^{[189]}$ exploited a chip-integrated biosensor array to realize the multiplexed detection of cancer biomarkers. Figure 9 (a) shows the design of a silicon microchip with 32 active microring sensors arranged in an array for simultaneously detecting eight different analytes in a fully automated antibody-based sandwich assay. Through the layer-by-layer signal enhancement strategy shown in Figure 9 (b), a real-time measurement of resonance wavelength shifts was plot during a multiplexed biomarker assay from a human serum sample (Figure 9 (c)). This work is highly effective and extremely useful in personalized cancer diagnostics. Later, Y.J. Chen et al ${ }^{[191]}$ demonstrated the integration of microbead WGM sensors with microfluidics to detect various biomarkers, which has sensitivity down to with nanomolar level (Figure 10). The microfluidic chip is designed to guide sample loading and control the flow rate. Through patterning the microfluidic device, microfluidic channels are also able to both release and trap different kinds of cells, which opens the possibility of realizing the sensing of multiple biomarkers. In this system, the drug solutions were continuously pumped into the microfluidic chip and the analyte was captured by the antibody functionalized microbead surface, thus enabling label-free biodetection. This work shows the great potential of combining precise microfluidic control with highly sensitive optical sensing devices to realize label-free and real-time biosensing with high sensitivity and accuracy. We can see that microfluidic chips play an important role in combining the reaction and detection process in a highly integrated way. 


\section{WILEY-VCH}

(a)

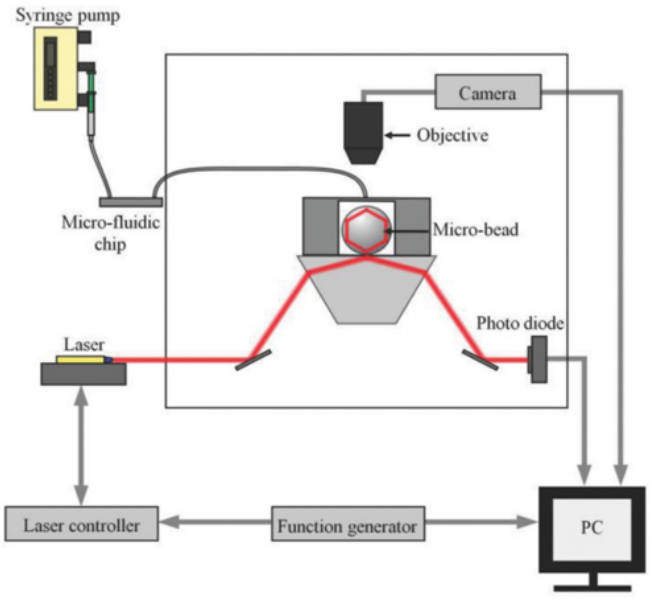

(b)

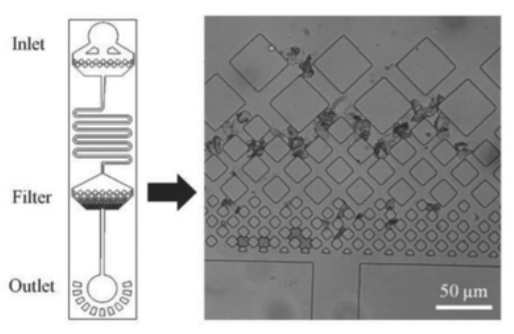

(c)

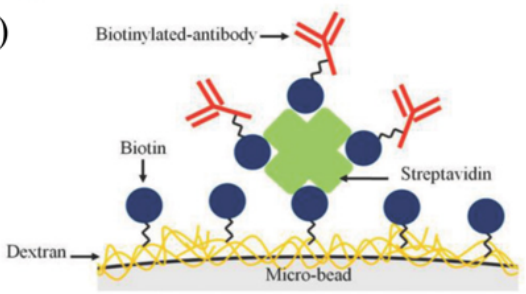

Figure 10. Microfluidic integrated WGMB. (a) WGMB system schematic. (b) Left: Microfluidic chip. Right: The Jurkat cells trapped in the microfluidic device. (c) Illustration of surface functionalization. Reproduced from ref. ${ }^{[191]}$ with permission of John Wiley and Sons.

\subsection{Temperature sensing}

Temperature variation can lead to mode shifting in WGM resonators through inducing a change of refractive index or size of the microcavity. Therefore, it is favorable to use materials with large thermal-optic and thermal expansion coefficient as the microcavity. Polydimethylsiloxane (PDMS) ${ }^{[192,193]}$, polymethyl methacrylate (PMMA) ${ }^{[194,195]}$, cholesteric liquid crystal (CLC) $[21,140]$, doped silica glass ${ }^{[196]}$ are some common choices for the resonator. Different geometries for the WGM resonators have been proposed, including microrings ${ }^{[87]}$, microdisks ${ }^{[197]}$, microspheres ${ }^{[198]}$, microtoroids ${ }^{[192]}$, microdroplets ${ }^{[147]}$ and microbubbles ${ }^{[164,195]}$. Recently, many researchers have devoted their efforts into building integrated sensing devices. L. Wan et al ${ }^{[87]}$ developed a chip-scale temperature sensor based on dye-doped polymer microring resonator. The organic dye R6G-doped polymer was deposited on the microring as a gain medium, which reduced the spectral linewidth and improved the detection limit. This active resonator-based temperature sensor enabled a simpler measurement setup with free-space optics. They characterized the spectral characteristics, lasing threshold and photostability to 


\section{WILEY-VCH}

validate the performance of this temperature sensor. X. Chen et al ${ }^{[199]}$ proposed a packaged droplet microresonator and demonstrated its application in thermal sensing (Figure 11). They simulated the regime of quasi-droplet resonators through Mie theory and experimentally demonstrated the thermal sensing with a sensitivity up to $205.3 \mathrm{pm} /{ }^{\circ} \mathrm{C}$. The packaged design offers the advantages of high compactness and facile fabrication. The capability of operating through free-space optics also has great potential in integrated photonic sensing devices. There is also report in developing wireless temperature sensor with a packaged microtoroid resonator ${ }^{[200]}$. A UV curable low-index polymer was used to package the resonator with a fiber-taper waveguide, which ensures the long-term stability of the device. Using this system, both realtime temperature and temperature distribution in selected areas can be measured. Moreover, a user-friendly interface with wireless module and customized app was built. This work has shown the capability of integrating WGM sensors with wireless interfaces for remote measurement and analysis of sensing signals.
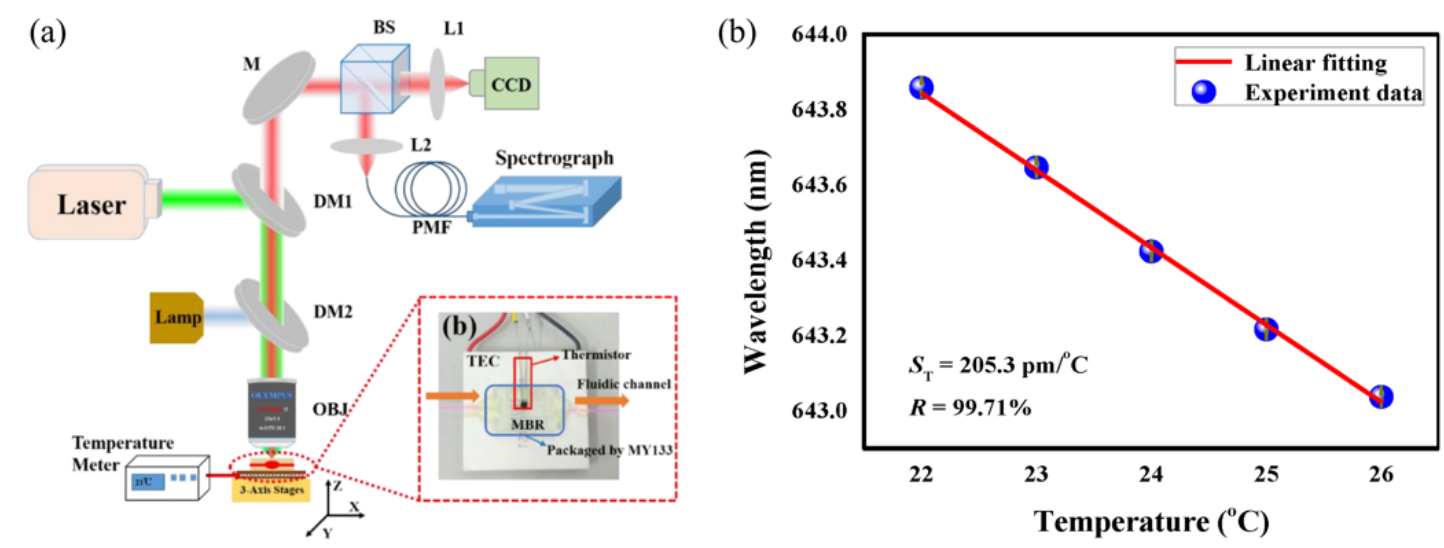

Figure 11. (a) The experimental setup of the WGM thermal sensor. (b) The resonance shifts as a linear function of the temperature. Reproduced from ref. ${ }^{[199]}$ with permission of Multidisciplinary Digital Publishing Institute. 


\section{WILEY-VCH}

In Table 3, we summarize the temperature sensing sensitivity of different types of WGM resonators in recent papers. The sensitivity shown in the table is achieved in the corresponding temperature detection range, as also listed in Table 3. Note that the detection range represents the experimental data in each paper instead of the maximum detection range for each category. Generally, the liquid resonators can achieve higher sensitivity than solid ones, but their sensing range is compromised. Therefore, the type of microresonator needs to be selected according to the specific sensing requirements and conditions.

\begin{tabular}{|c|c|c|c|}
\hline \multicolumn{2}{|c|}{ Type of microresonators $^{2}$} & $\begin{array}{c}\text { Sensitivity } \\
\text { (pm/ C) }\end{array}$ & Detection \\
range
\end{tabular}

Table 3. Sensitivity comparison of WGM temperature sensors based on different microresonators.

\subsection{Humidity sensing}




\section{WILEY-VCH}

The refractive index and size of the resonators can also change when absorbing water vapor ${ }^{[204]}$. As a result, WGM resonators can also be exploited as humidity sensors. Efforts have been paid to improve the sensitivity through optimizing the size and the material of the microcavity ${ }^{[205]}$. Many researchers have reported microspheres as the resonators to successfully realize the sensing of relative humidity $(\mathrm{RH})^{[206,207]}$. M. Eryürek et al ${ }^{[208]}$ utilized SU-8 polymer microdisk, which is a material that has high interaction with water vapor and also easy-to-fabricate, to make an integrated humidity sensor (Figure 12 (a)). This integrated sensor with microdisk resonator and optical waveguide was fabricated through standard single-step UV photolithography. The chip was placed inside a gas chamber to test relative humidity (RH) from 0 to $50 \%$. A tunable laser light is coupled to SU-8 waveguides through end-face coupling method and a photodiode is placed at the other end of the waveguide to collect the transmission signal. Then, the shift in the transmission spectra resulted from both refractive index change and radius change of the resonator can be measured as the sensing signal. The experimental results show a sensitivity of $108 \mathrm{pm} / \% \mathrm{RH}$ between 0 and $1 \% \mathrm{RH}$ and good sensing response for $\mathrm{RH}$ values up to $50 \%$. This device has the advantages of high sensitivity as well as easy fabrication. Microbottles which have high Q-factor and good performance are also exploited in humidity sensing applications. M. A. Md Johari et al ${ }^{[209]}$ fabricated a microbottle resonator (MBR) through "soften-and-compress" method. The MBR was optically excited and proved to have a Q-factor of $10^{4}$. They evaluated the humidity sensing ability of both MBR and no-MBR microfiber, which showed the superiority of MBR over no-MBR in both sensitivity and linearity. The effectiveness of a liquid droplet acting as a humidity sensor has been exemplified by L. Labrador-Páez et al ${ }^{[210]}$. They utilized a droplet made of glycerol doped with fluorescent material as the resonator (shown in Figure 12 (c)). The droplet resonator was formed at the tip of a modified optical fiber and the fluorescent doping makes it possible to directly observe WGMs without coupling to a tapered optical fiber. Since the refractive index and radius of the 


\section{WILEY-VCH}

droplet will change with ambient humidity, this device was implemented as a highly effective humidity sensor with high sensitivity of $10^{-3}$ per relative humidity percent.

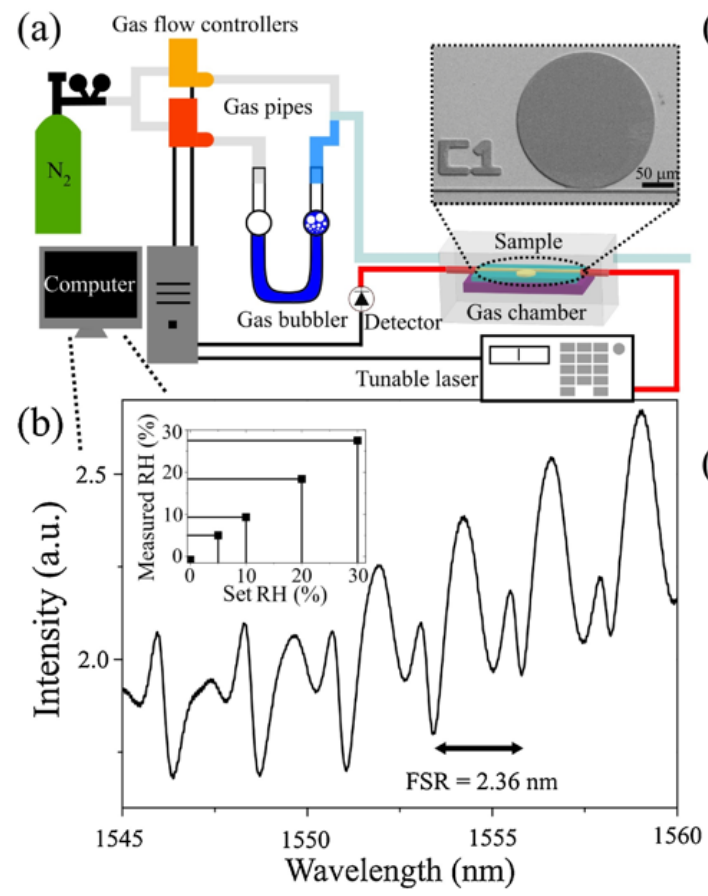

(c)
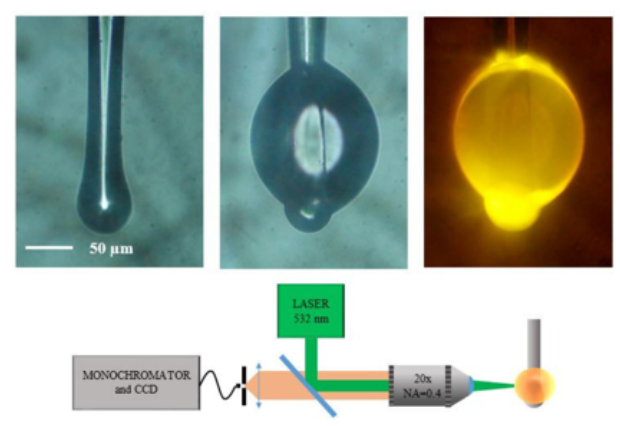

(d)

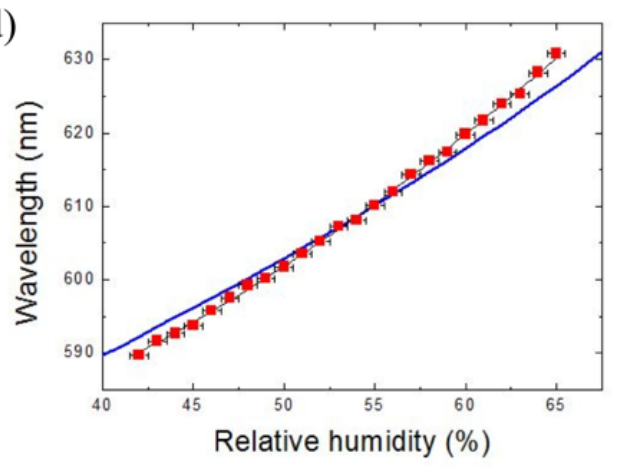

Figure 12. (a) Experimental setup of the integrated humidity sensor. (b) One exemplary transmission spectrum. Reproduced from ref. ${ }^{[208]}$ with permission of Elsevier. (c) Image of the liquid resonator made in the tip of the modified optical fiber and experimental setup. (d) Calibration of the variation of the wavelength of a WGM peak with increasing RH (red squares) and estimation of the displacement of the wavelength of the WGM peak from the expected variation of the refractive index and the radius of the resonator with RH (blue line). Reproduced from ref. ${ }^{[210]}$ with permission of The Optical Society.

\subsection{Pressure sensing}

The spectral shift response of whispering gallery modes with regards to the pressure or strain has also led to many sensing applications. Several investigations have been carried out concerning the effects of pressure on the behavior of microsphere resonators. H. P. Wagner et al $^{[211]}$ specifically studied the effects of uniaxial stress on polar WGM and pointed out that the WGM resonance will reveal a blue shift when the pressure increases. The magnitude of this shift depends on the radial mode number n. Moreover, L. L. Martin et al ${ }^{[212]}$ simulated that the 


\section{WILEY-VCH}

average sensitivity of silicate microsphere resonators can reach $6.5 \times 10^{-4} \mathrm{GPa}^{-1}$, which presents great advantages compared to conventional pressure sensor. More recently, A. Bianchetti et al ${ }^{[213]}$ analyzed a refractometry based pressure sensor with spherical glass WGM microresonators (Figure 13). The pressure sensing ability has been characterized by measuring the WGM shifts caused by dynamic sinusoidal pressure variations between $\pm 1.8 \mathrm{kPa}$. It is also verified that adopting smaller radius of resonator and exciting TM-polarized modes instead of TE-polarized modes can lead to higher sensitivity. Hollow resonators have also been utilized as an effective way for pressure sensing owing to their particularly high sensitivity towards sensing air pressure changes. The microbubble resonator proposed by R. Madugani et al ${ }^{[214]}$ is capable of measuring pressure with a detection limit of $2 \times 10^{-4} \mathrm{MPa}$. They applied the Pound-Drever-Hall (PDH) frequency locking technique to reduce the environmental noise. In this system, the fibercoupled tunable laser source was split into two beams. One was used in the feedback loop for the PDH locking while the other was firstly phase modulated, then coupled to the microbubble and detected through a photodiode. This system was also proved to have no hysteresis and longterm stability, which has great advantages in pressure sensing. Y. Yang et al ${ }^{[23]}$ fabricated a microbubble resonator with ultra-thin wall $(\sim 500 \mathrm{~nm})$ and extremely high Q-factor $\left(>5 \times 10^{7}\right)$, which allows for pressure sensing with a resolution of 0.17 mbar. Techniques including the incorporation of membranes ${ }^{[215,216]}$ and orthogonal demodulation Pound-Drever-Hall ${ }^{\text {[217] }}$ have also been developed to increase the sensitivity as well as decreasing the detection limit of pressure sensing. 


\section{WILEY-VCH}

(a)

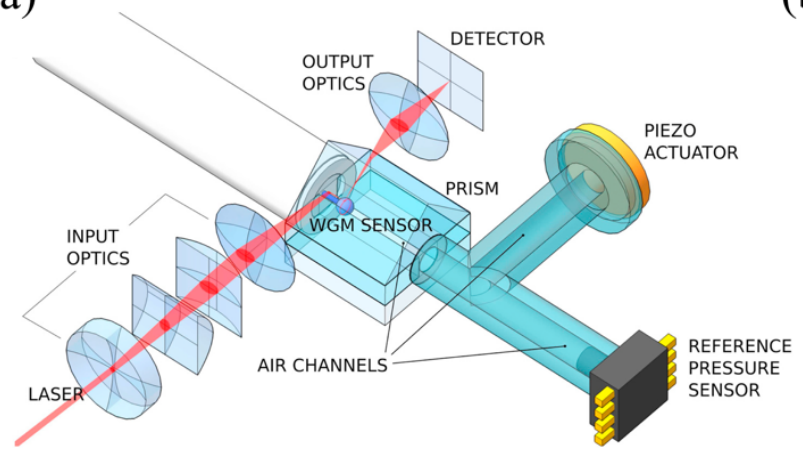

(b)

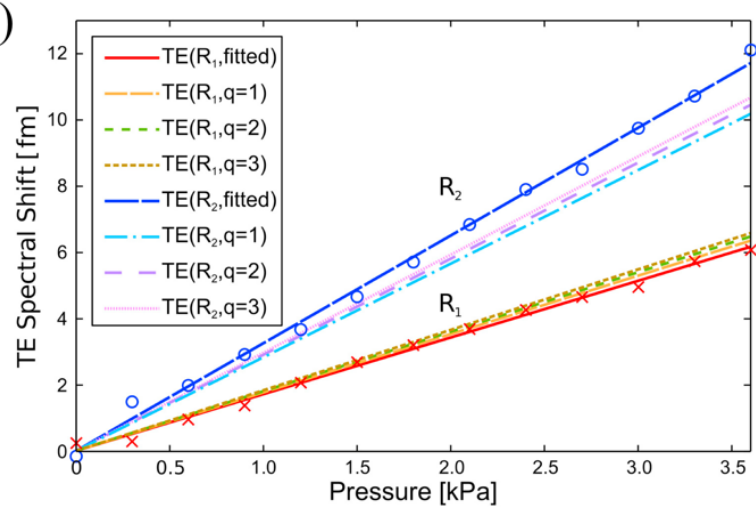

Figure 13. (a) Experimental setup of the WGM pressure sensing platform. (b) Spectral shift versus pressure for two spheres of radii $\mathrm{R}_{1}=127 \mu \mathrm{m}$ and $\mathrm{R}_{2}=89 \mu \mathrm{m}$. Reproduced from ref. ${ }^{[213]}$ with permission of Elsevier.

\subsection{Ultrasound sensing}

The high Q-factor of optical WGM resonators also makes it a promising approach to realize high frequency ultrasound detection, which enables non-invasive medical imaging as well as structural diagnosis of biological samples ${ }^{[218-220]}$. Over these years, various microfluidic optical resonators have been exploited for highly sensitive ultrasound detection in miniaturized sensing devices. The most commonly developed types here are micro-ring resonator-based ultrasound sensors ${ }^{[221-223]}$. H. Li et al designed a transparent ultrasound detector based on a polymeric optical micro-ring resonator ${ }^{[224]}$. It enables ultrasound detection with a high sensitivity down to an estimated noise-equivalent pressure (NEP) of 6.8 Pa and an ultra-broad bandwidth of 140 MHz. Figure 14 (a) illustrates the packaged ultrasound detector. Two tapered fiber were exploited to couple light in and out of the polymer waveguide with a 90-degree bend. This method simplifies the packaging procedure and has the potential to realize high coupling efficiency owing to the better mode matching between the tapered fiber and the bus waveguide. Figure 14 (b) shows the experimental setup of the photoacoustic microscopy system. The miniaturization and optical transparency of microring resonator was able to be utilized for microscopy in reflection mode, which makes it compatible with various imaging modalities ${ }^{[225]}$. 


\section{WILEY-VCH}

(a)

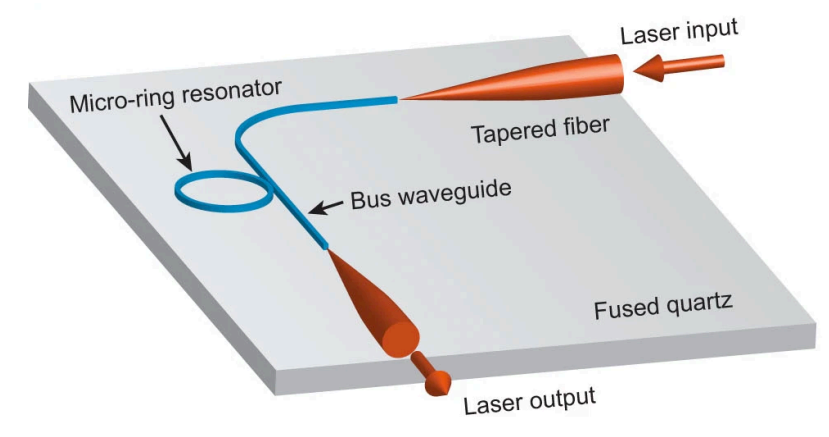

(b)

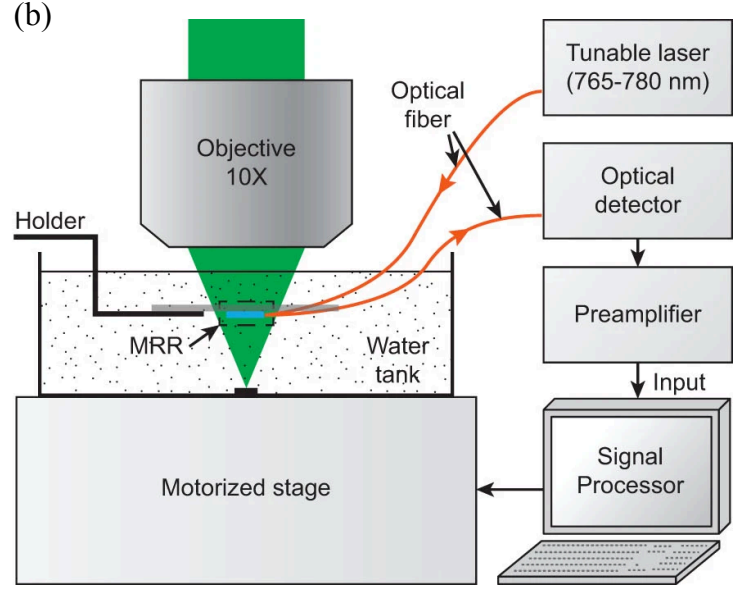

Figure 14. (a) Schematic illustrations of the packaged micro-ring ultrasonic detector. (b) Experimental setup of the photoacoustic microscopy system. Reproduced from ref. ${ }^{[224]}$ with permission of The Optical Society.

More recently, optical microbubble resonators were also studied as a promising tool for highprecision ultrasound detector. J. Pan et al presented a novel ultrasound detection approach based on high-Q optical microbubble resonators combined with a digital optical frequency comb technique ${ }^{[226]}$. This hollow microbubble resonator with an ultrathin wall will enhance the sensitivity to a NEP as low as $4.4 \mathrm{mPa} / \sqrt{\mathrm{Hz}}$ at $165 \mathrm{kHz}$. In another work, the microbubble resonator serve both as the acoustic transducer and as the microvial for sample preservation ${ }^{[227]}$. The combination with microfluidic design enabled photoacoustic inspection without the need for impedance-matching media. In summary, the small size and high sensitivity of optical resonators have made it a great choice for ultrasound detection, which has great potential in clinical endoscopic systems.

To date, WGM based sensing techniques have expanded to cover a range of application areas apart from those mentioned above. WGM resonators have also been exploited as sensors for displacement ${ }^{[228-230]}$, refractive index ${ }^{[231-233]}$, electric fields ${ }^{[234,235]}$, magnetic fields ${ }^{[236-240]}$, liquid concentration ${ }^{[241]}$ and gas sensing ${ }^{[242-244]}$. We anticipate that WGM resonators will play more and more significant roles in multiple sensing application areas. 


\section{WILEY-VCH}

\section{Conclusion and future prospects}

In this paper, we have reviewed several sensing mechanisms of WGM sensors and summarized different geometry types of WGM resonators. We also introduced the techniques that can be exploited to increase the sensitivity of these resonators. It is worth noting that there is a rapid progress in the development of integrated systems for portable and multiplexed sensing. In fact, a number of new WGM sensors have already emerged, as summarized in Section 3, covering various coupling techniques, packaging schemes and integration designs. Many researchers have investigated the combination schemes for microfluidic chips with WGM resonators to develop lab-on-chip devices for sensing applications. Thus, we have highlighted the recent trend of integrating WGM sensors with microfluidics and compared the sensing performance, together with different resonator designs and various integration schemes (see Table 2). This review paper provides a useful guideline for integrating optical sensing techniques with microfluidic systems to perform both the handling and detection of samples, making full use of the liquid manipulation as well as multiple sample analysis capabilities of microfluidic chips and high detection sensitivity of WGM resonators. In the last section, we have discussed the applications of these integrated WGM sensors in different sensing areas.

Nevertheless, current development of integrated microfluidic-based WGM sensors still faces several challenges: (i) The elimination of environmental disturbance including thermal fluctuations and spectroscopic noise still needs to be improved for all WGM sensors. Further enhancement on sensitivity and accuracy of the device is still needed. (ii) The design of a more robust and stable packaging system while maintaining the ability of bio-chemical sensing can be further explored. Despite the current development on packaging methods, few of them can achieve both high system stability and high sensitivity, together with the ease of fabrication.

(iii) The multiplexing ability of WGM sensors are highly desired nowadays. Currently, the 


\section{WILEY-VCH}

coupling techniques are still not optimized to build a robust device with multiple WGM resonators in single chip platform while possessing the ultrahigh sensitivity ${ }^{[123]}$. There is still much room for realizing multiplexed detection using an array of WGM resonators, which is of great importance in many biological applications. (iv) The large-scale optical integration of onchip resonators remains a challenge, which is of great importance to promote their widespread field application in portable and on-site detections ${ }^{[118]}$. More studies should be carried out on optimizing material properties and fabrication schemes of the WGM resonators to facilitate large-scale integration. (v) To date, the research community has not established a general standard for testing these devices. Thus, the establishment of a reference standard such as ELISA for integrated WGM biosensors ${ }^{[19]}$ will accelerate adoption rate by prospective users. (vi) Most of the current microfluidic-based WGM sensors focus more on the design of the packaging schemes for the microresonators rather than the whole automation process from solution loading to sample detection. However, the concept of full integration represents an allin-one automated system with the abilities to conduct controllable sample loading, reaction, detection and analysis in one device, which has not yet been realized in the current development of WGM sensing systems.

To summarize, we believe that the key challenge and opportunity here is to make full use of the advantages of both microfluidic techniques and WGM microresonators. In addition to further improving the Q-factor of WGM microresontors, we also should pay more attention to utilizing the current microfluidic integration techniques to increase the stability and reduce the size of the sensing device. Most importantly, the convenient sample handling and multiplexed detection abilities of microfluidic technologies should be exploited to promote the practical applications of microfluidic-based WGM sensors. One can readily see that if more efforts are put into the development of fully integrated WGM sensors, all-in-one point-of-care diagnostic 


\section{WILEY-VCH}

devices with high detection sensitivity as well as high robustness will be realized in the near future.

\section{Acknowledgment}

This work is supported under the PROCORE - France/Hong Kong Joint Research Scheme (FCUHK402/19). The authors also would like to acknowledge the support provide by the Research Grants Council (Project No.: 14207419, 14210517, N-CUHK407/16, AoE/P-0/12) and The Innovation and Technology Council of the Hong Kong Special Administrative Region (Project No.: ITS061/18, GHP/014/13SZ). This project has received funding from the European Union's Horizon 2020 research and innovation programme under grant agreement No. 798916.

Received: ((will be filled in by the editorial staff))

Revised: ((will be filled in by the editorial staff)) Published online: ((will be filled in by the editorial staff)) 


\section{WILEY-VCH}

\section{References}

[1] Y. N. Zhang, T. Zhou, B. Han, A. Zhang, Y. Zhao, Nanoscale, 10, 13832-13856 (2018).

[2] J. R. Askim, M. Mahmoudi, K. S. Suslick, Chem Soc Rev, 42, 8649-8682 (2013).

[3] A.-K. Mahlein, E.-C. Oerke, U. Steiner, H.-W. Dehne, European Journal of Plant Pathology, 133, 197-209 (2012).

[4] Y. Fang, R. P. Ramasamy, Biosensors (Basel), 5, 537-561 (2015).

[5] M. Li, S. K. Cushing, N. Wu, Analyst, 140, 386-406 (2015).

[6] F. Long, A. Zhu, H. Shi, Sensors (Basel), 13, 13928-13948 (2013).

[7] M. Saleem, K. H. Lee, RSC Advances, 5, 72150-72287 (2015).

[8] K. Narsaiah, S. N. Jha, R. Bhardwaj, R. Sharma, R. Kumar, J Food Sci Technol, 49, 383-406 (2012).

[9] N. Khansili, G. Rattu, P. M. Krishna, Sensors and Actuators B: Chemical, 265, 35-49 (2018).

[10] J. F. Masson, ACS Sens, 2, 16-30 (2017).

[11] H. H. Nguyen, J. Park, S. Kang, M. Kim, Sensors (Basel), 15, 10481-10510 (2015).

[12] Y. Zhao, H. Zhao, R.-q. Lv, J. Zhao, Optics and Lasers in Engineering, 117, 7-20 (2019).

[13] Y.-n. Zhang, Y. Zhao, R.-q. Lv, Sensors and Actuators A: Physical, 233, 374-389 (2015).

[14] H. Qu, M. Skorobogatiy, Sensors and Actuators B: Chemical, 161, 261-268 (2012).

[15] S. Sang, Y. Wang, Q. Feng, Y. Wei, J. Ji, W. Zhang, Crit Rev Biotechnol, 36, 465-481 (2016).

[16] A. A. Savchenkov, A. B. Matsko, V. S. Ilchenko, L. Maleki, Optics express, 15, 67686773 (2007).

[17] J. Ward, O. Benson, Laser \& Photonics Reviews, 5, 553-570 (2011).

[18] I. Brevik, S. Å. Ellingsen, Physical Review A, 81, (2010).

[19] J. Su, Sensors (Basel), 17, (2017).

[20] F. Vollmer, L. Yang, Nanophotonics, 1, 267-291 (2012).

[21] L. Zhao, Y. Wang, Y. Yuan, Y. Liu, S. Liu, W. Sun, J. Yang, H. Li, Optics Communications, 402, 181-185 (2017).

[22] A. Petermann, T. Hildebrandt, U. Morgner, B. Roth, M. Meinhardt-Wollweber, Sensors, 18, (2018).

[23] Y. Yang, S. Saurabh, J. M. Ward, S. Nic Chormaic, Opt Express, 24, 294-299 (2016).

[24] J. Su, ACS Photonics, 2, 1241-1245 (2015).

[25] L. Shao, X. F. Jiang, X. C. Yu, B. B. Li, W. R. Clements, F. Vollmer, W. Wang, Y. F. Xiao, Q. Gong, Adv Mater, 25, 5616-5620 (2013).

[26] J. Su, A. F. Goldberg, B. M. Stoltz, Light Sci Appl, 5, e16001 (2016).

[27] V. R. Dantham, S. Holler, C. Barbre, D. Keng, V. Kolchenko, S. Arnold, Nano Lett, 13, 3347-3351 (2013).

[28] Y. Zheng, Z. Wu, P. Ping Shum, Z. Xu, G. Keiser, G. Humbert, H. Zhang, S. Zeng, X. Quyen Dinh, Opto-Electronic Advances, 1, 18001501-18001510 (2018).

[29] E. Kim, M. D. Baaske, F. Vollmer, Lab on a Chip, 17, 1190-1205 (2017).

[30] M. R. Foreman, J. D. Swaim, F. Vollmer, Advances in Optics and Photonics, 7, (2015).

[31] Z. Liao, Y. Zhang, Y. Li, Y. Miao, S. Gao, F. Lin, Y. Deng, L. Geng, Biosens Bioelectron, 126, 697-706 (2019).

[32] O. Yavas, S. S. Acimovic, J. Garcia-Guirado, J. Berthelot, P. Dobosz, V. Sanz, R. Quidant, ACS Sens, 3, 1376-1384 (2018). 


\section{WILEY-VCH}

[33] G. M. Whitesides, Nature, 442, 368-373 (2006).

[34] Y. Wang, S. Liu, T. Zhang, H. Cong, Y. Wei, J. Xu, Y.-P. Ho, S.-K. Kong, H.-P. Ho, Lab on a Chip, 19, 3870-3879 (2019).

[35] M. Tang, X. Huang, Q. Chu, X. Ning, Y. Wang, S. K. Kong, X. Zhang, G. Wang, H. P. Ho, Lab Chip, 18, 1452-1460 (2018).

[36] K. E. Bates, H. Lu, Biophys J, 110, 1684-1697 (2016).

[37] J. P. Lafleur, A. Jonsson, S. Senkbeil, J. P. Kutter, Biosens Bioelectron, 76, 213-233 (2016).

[38] T. Lu, H. Lee, T. Chen, S. Herchak, J. H. Kim, S. E. Fraser, R. C. Flagan, K. Vahala, Proc Natl Acad Sci U S A, 108, 5976-5979 (2011).

[39] J. Knittel, J. D. Swaim, D. L. McAuslan, G. A. Brawley, W. P. Bowen, Scientific Reports, 3, (2013).

[40] L. He, S. K. Ozdemir, J. Zhu, W. Kim, L. Yang, Nat Nanotechnol, 6, 428-432 (2011).

[41] A. Bozzola, S. Perotto, F. De Angelis, Analyst, 142, 883-898 (2017).

[42] F. Vollmer, S. Arnold, Nat Methods, 5, 591-596 (2008).

[43] S. Arnold, M. Khoshsima, I. Teraoka, S. Holler, F. Vollmer, Optics letters, 28, 272274 (2003).

[44] F. Vollmer, S. Arnold, D. Keng, Proceedings of the National Academy of Sciences, 105, 20701-20704 (2008).

[45] J. W. Silverstone, S. McFarlane, C. P. K. Manchee, A. Meldrum, Optics express, 20, 8284-8295 (2012).

[46] M. R. Foreman, F. Vollmer, New Journal of Physics, 15, (2013).

[47] Y. Shen, J.-T. Shen, Physical Review A, 85, (2012).

[48] Y. D. Chiu, C. W. Wu, C. C. Chiang, Sensors (Basel), 17, (2017).

[49] G. Guan, S. Arnold, V. Otugen, AIAA Journal, 44, 2385-2389 (2006).

[50] N. Sung Hyun, Y. Shizhuo, IEEE Photonics Technology Letters, 17, 2391-2393 (2005).

[51] M. D. Baaske, M. R. Foreman, F. Vollmer, Nature Nanotechnology, 9, 933-939 (2014).

[52] M. D. Baaske, F. Vollmer, Nature Photonics, 10, 733-739 (2016).

[53] B.-Q. Shen, X.-C. Yu, Y. Zhi, L. Wang, D. Kim, Q. Gong, Y.-F. Xiao, Physical Review Applied, 5, (2016).

[54] T. J. Kippenberg, Nature Photonics, 4, 9-10 (2010).

[55] Y.-C. Liu, Y.-F. Xiao, B.-B. Li, X.-F. Jiang, Y. Li, Q. Gong, Physical Review A, 84, (2011).

[56] S. K. Ozdemir, J. Zhu, X. Yang, B. Peng, H. Yilmaz, L. He, F. Monifi, S. H. Huang, G. L. Long, L. Yang, Proc Natl Acad Sci U S A, 111, E3836-3844 (2014).

[57] B. B. Li, W. R. Clements, X. C. Yu, K. Shi, Q. Gong, Y. F. Xiao, Proc Natl Acad Sci U S A, 111, 14657-14662 (2014).

[58] J. Zhu, S. K. Ozdemir, Y.-F. Xiao, L. Li, L. He, D.-R. Chen, L. Yang, Nature Photonics, 4, 46-49 (2009).

[59] A. J. Qavi, A. L. Washburn, J. Y. Byeon, R. C. Bailey, Anal Bioanal Chem, 394, 121135 (2009).

[60] C. Klusmann, J. Oppermann, P. Forster, C. Rockstuhl, H. Kalt, ACS Photonics, 5, 2365-2373 (2018).

[61] M. Zhang, G. Wu, Optics Communications, 403, 68-72 (2017).

[62] M. Zhang, G. Wu, D. Chen, Applied Optics, 54, (2015).

[63] P. Peng, Y.-C. Liu, D. Xu, Q.-T. Cao, G. Lu, Q. Gong, Y.-F. Xiao, Physical Review Letters, 119, (2017).

[64] N. Thakkar, M. T. Rea, K. C. Smith, K. D. Heylman, S. C. Quillin, K. A. Knapper, E. H. Horak, D. J. Masiello, R. H. Goldsmith, Nano Letters, 17, 6927-6934 (2017). 


\section{WILEY-VCH}

[65] J. Zhang, X. Jin, P. I. Morales-Guzman, X. Yu, H. Liu, H. Zhang, L. Razzari, J. P. Claverie, ACS Nano, 10, 4496-4503 (2016).

[66] Q. Zhu, C. Xu, D. Wang, B. Liu, F. Qin, Z. Zhu, Y. Liu, X. Zhao, Z. Shi, Journal of Materials Chemistry C, 7, 2710-2716 (2019).

[67] E. Ozgur, K. E. Roberts, E. O. Ozgur, A. N. Gin, J. R. Bankhead, Z. Wang, J. Su, Analytical Chemistry, 91, 11872-11878 (2019).

[68] J. D. Swaim, J. Knittel, W. P. Bowen, Applied Physics Letters, 102, (2013).

[69] S. I. Shopova, R. Rajmangal, Y. Nishida, S. Arnold, Rev Sci Instrum, 81, 103110 (2010).

[70] W. Chen, S. Kaya Ozdemir, G. Zhao, J. Wiersig, L. Yang, Nature, 548, 192-196 (2017).

[71] J. Wiersig, Physical Review Letters, 112, (2014).

[72] M. Brandstetter, M. Liertzer, C. Deutsch, P. Klang, J. Schöberl, H. E. Türeci, G.

Strasser, K. Unterrainer, S. Rotter, Nature Communications, 5, (2014).

[73] X.-F. Jiang, C.-L. Zou, L. Wang, Q. Gong, Y.-F. Xiao, Laser \& Photonics Reviews, 10, 40-61 (2016).

[74] G. Zhao, Ş. K. Özdemir, T. Wang, L. Xu, E. King, G.-L. Long, L. Yang, Science Bulletin, 62, 875-878 (2017).

[75] K. Wang, G. Li, S. Wang, S. Liu, W. Sun, C. Huang, Y. Wang, Q. Song, S. Xiao, Adv Mater, 30, e1801481 (2018).

[76] N. Zhang, Z. Gu, S. Liu, Y. Wang, S. Wang, Z. Duan, W. Sun, Y.-F. Xiao, S. Xiao, Q. Song, Optica, 4, (2017).

[77] S. Rosenblum, Y. Lovsky, L. Arazi, F. Vollmer, B. Dayan, Nat Commun, 6, 6788 (2015).

[78] M.-Y. Ye, X.-M. Lin, Optics Express, 25, (2017).

[79] Y. Yang, R. Madugani, S. Kasumie, J. M. Ward, S. Nic Chormaic, in Exploring the

World with the Laser, (Eds: D. Meschede, T. Udem, T. Esslinger), Springer International

Publishing, Cham 2018, 629-646.

[80] H. Jayamohan, V. Romanov, H. Li, J. Son, R. Samuel, J. Nelson, B. K. Gale, in Molecular Diagnostics, 2017, 197-217.

[81] I. Rodriguez-Ruiz, T. N. Ackermann, X. Munoz-Berbel, A. Llobera, Anal Chem, 88, 6630-6637 (2016).

[82] G. C. Righini, S. Berneschi, A. Cosci, D. Farnesi, A. Giannetti, G. N. Conti, S. Pelli, S. Soria, Optical Sensors, (2017).

[83] Y. Wang, H. Li, L. Zhao, B. Wu, S. Liu, Y. Liu, J. Yang, Optics \& Laser Technology, 86, 61-68 (2016).

[84] M. R. Foreman, S. Avino, R. Zullo, H. P. Loock, F. Vollmer, G. Gagliardi, The European Physical Journal Special Topics, 223, 1971-1988 (2014).

[85] J. M. Ward, N. Dhasmana, S. Nic Chormaic, The European Physical Journal Special Topics, 223, 1917-1935 (2014).

[86] K. Y. Fong, M. Poot, H. X. Tang, Nano Lett, 15, 6116-6120 (2015).

[87] L. Wan, H. Chandrahalim, C. Chen, Q. Chen, T. Mei, Y. Oki, N. Nishimura, L. J.

Guo, X. Fan, Applied Physics Letters, 111, (2017).

[88] M. K. Park, J. S. Kee, J. Y. Quah, V. Netto, J. Song, Q. Fang, E. M. La Fosse, G.-Q. Lo, Sensors and Actuators B: Chemical, 176, 552-559 (2013).

[89] R. An, G. Wang, W. Ji, W. Jiao, M. Jiang, Y. Chang, X. Xu, N. Zou, X. Zhang, Opt Lett, 43, 3901-3904 (2018).

[90] W. Jiao, G. Wang, Z. Ying, Y. Zou, H. P. Ho, T. Sun, Y. Huang, X. Zhang, Opt Lett, 41, 2652-2655 (2016).

[91] W. Xu, Y. Wang, W. Jiao, F. Wang, X. Xu, M. Jiang, H. P. Ho, G. Wang, Opt Lett, 44, 3226-3229 (2019). 


\section{WILEY-VCH}

[92] X. Xu, Y. Dong, G. Wang, W. Jiao, Z. Ying, H.-p. Ho, X. Zhang, IEEE Photonics Journal, 10, 1-7 (2018).

[93] Z. Hao, L. Zhang, A. Gao, W. Mao, X. Lyu, X. Gao, F. Bo, F. Gao, G. Zhang, J. Xu, Science China Physics, Mechanics \& Astronomy, 61, (2018).

[94] S. Liu, W. Sun, Y. Wang, X. Yu, K. Xu, Y. Huang, S. Xiao, Q. Song, Optica, 5, (2018).

[95] Z. Wang, S. Yuan, G. Dong, R. Wang, L. Chen, X. Wu, X. Zhang, Optics Letters, 44, (2019).

[96] W. Li, H. Zhang, J. Liu, J. Lin, X. Xue, X. Zhang, X. Xu, A. Huang, Z. Xiao, Journal of the Optical Society of America B, 34, (2017).

[97] S. M. Harazim, V. A. Bolaños Quiñones, S. Kiravittaya, S. Sanchez, O. G. Schmidt, Lab on a Chip, 12, (2012).

[98] M. Frenkel, Z. Guo, Journal of Polymer Science Part B: Polymer Physics, 54, 11181124 (2016).

[99] Q. Lu, X. Chen, L. Fu, S. Xie, X. Wu, Nanomaterials (Basel), 9, (2019).

[100] A. L. Washburn, L. C. Gunn, R. C. Bailey, Analytical chemistry, 81, 9499-9506 (2009).

[101] R. M. Graybill, C. S. Para, R. C. Bailey, Anal Chem, 88, 10347-10351 (2016).

[102] E. H. Mordan, J. H. Wade, Z. S. B. Wiersma, E. Pearce, T. O. Pangburn, A. W. deGroot, D. M. Meunier, R. C. Bailey, Anal Chem, 91, 1011-1018 (2019).

[103] Y. Wang, N. Zhang, Z. Jiang, L. Wang, Y. Xiao, W. Sun, N. Yi, S. Liu, X. Gu, S. Xiao, Q. Song, Advanced Materials Technologies, 2, (2017).

[104] L. Kelemen, E. Lepera, B. Horváth, P. Ormos, R. Osellame, R. Martínez Vázquez, Lab on a Chip, 19, 1985-1990 (2019).

[105] S. Wu, Y. Guo, W. Wang, J. Zhou, Q. Zhang, Rev Sci Instrum, 90, 035004 (2019).

[106] C. F. Carlborg, K. B. Gylfason, A. Kaźmierczak, F. Dortu, M. J. Bañuls Polo, A.

Maquieira Catala, G. M. Kresbach, H. Sohlström, T. Moh, L. Vivien, J. Popplewell, G.

Ronan, C. A. Barrios, G. Stemme, W. van der Wijngaart, Lab Chip, 10, 281-290 (2010).

[107] G. A. Rodriguez, S. Hu, S. M. Weiss, Opt Express, 23, 7111-7119 (2015).

[108] V. Zamora, P. Lützow, M. Weiland, D. Pergande, Sensors, 13, 14601-14610 (2013).

[109] V. Zamora, P. Lützow, M. Weiland, D. Pergande, Optics Express, 21, (2013).

[110] M. La Notte, V. M. N. Passaro, Sensors and Actuators B: Chemical, 176, 994-1007 (2013).

[111] S. Lin, K. B. Crozier, ACS Nano, 7, 1725-1730 (2013).

[112] S. Panich, K. A. Wilson, P. Nuttall, C. K. Wood, T. Albrecht, J. B. Edel, Analytical Chemistry, 86, 6299-6306 (2014).

[113] C. Zhang, A. Cocking, E. Freeman, Z. Liu, S. Tadigadapa, Sci Rep, 7, 14965 (2017).

[114] T. Wienhold, S. Kraemmer, S. F. Wondimu, T. Siegle, U. Bog, U. Weinzierl, S.

Schmidt, H. Becker, H. Kalt, T. Mappes, S. Koeber, C. Koos, Lab on a Chip, 15, 3800-3806 (2015).

[115] X. Gao, J. Li, Z. Hao, F. Bo, C. Hu, J. Wang, Z. Liu, Z.-Y. Li, G. Zhang, J. Xu, Journal of Applied Physics, 121, (2017).

[116] L. Wang, S.-X. Zhang, Q. Song, Q. Gong, Y.-F. Xiao, Optica, 3, (2016).

[117] A. G. Griffith, R. K. Lau, J. Cardenas, Y. Okawachi, A. Mohanty, R. Fain, Y. H. Lee, M. Yu, C. T. Phare, C. B. Poitras, A. L. Gaeta, M. Lipson, Nat Commun, 6, 6299 (2015).

[118] K. A. Knapper, K. D. Heylman, E. H. Horak, R. H. Goldsmith, Advanced Materials, 28, 2945-2950 (2016).

[119] T. Grossmann, M. Hauser, T. Beck, C. Gohn-Kreuz, M. Karl, H. Kalt, C. Vannahme, T. Mappes, Applied Physics Letters, 96, (2010). 


\section{WILEY-VCH}

[120] Y. Xuan, Y. Liu, L. T. Varghese, A. J. Metcalf, X. Xue, P.-H. Wang, K. Han, J. A. Jaramillo-Villegas, A. Al Noman, C. Wang, S. Kim, M. Teng, Y. J. Lee, B. Niu, L. Fan, J. Wang, D. E. Leaird, A. M. Weiner, M. Qi, Optica, 3, (2016).

[121] X. Zhang, A. M. Armani, Opt Express, 21, 23592-23603 (2013).

[122] Y. Z. Yan, Zou, C.L., Yan, S.B., Sun, F.W., Ji, Z., Liu, J., Zhang, Y.G., Wang, L., Xue, C.Y., Zhang, W.D. and Han, Z.F., Optics express, 19, 5753-5759 (2011).

[123] F. Monifi, S. K. Ozdemir, J. Friedlein, L. Yang, IEEE Photonics Technology Letters, 25, 1458-1461 (2013).

[124] Y.-Z. Yan, C.-L. Zou, S.-B. Yan, F.-W. Sun, J. Liu, C.-Y. Xue, Y.-G. Zhang, L. Wang, W.-D. Zhang, J.-J. Xiong, IEEE Photonics Technology Letters, 23, 1736-1738 (2011). [125] F. Bo, S. H. Huang, S. K. Ozdemir, G. Zhang, J. Xu, L. Yang, Opt Lett, 39, 1841-1844 (2014).

[126] R. Suzuki, T. Kato, T. Tetsumoto, T. Tanabe, AIP Advances, 5, (2015).

[127] L. Shi, T. Zhu, D. Huang, C. Liang, M. Liu, S. Liang, Opt Lett, 42, 167-170 (2017).

[128] R. Wang, M. Fraser, J. Li, X. Qiao, A. Wang, Opt Lett, 40, 308-311 (2015).

[129] X. Zhang, H. Bai, H. Pan, J. Wang, M. Yan, H. Xiao, T. Wang, IEEE Photonics Journal, 10, 1-8 (2018).

[130] X. Xu, X. Jiang, G. Zhao, L. Yang, Opt Express, 24, 25905-25910 (2016).

[131] A. I. Gubin, A. A. Barannik, N. T. Cherpak, I. A. Protsenko, S. Pud, A. Offenhausser, S. A. Vitusevich, IEEE Transactions on Microwave Theory and Techniques, 63, 2003-2009 (2015).

[132] S. M. Wildgen, R. C. Dunn, Biosensors (Basel), 5, 118-130 (2015).

[133] L. Luan, M. W. Royal, R. Evans, R. B. Fair, N. M. Jokerst, IEEE Sensors Journal, 12, 1794-1800 (2012).

[134] S. F. Wondimu, S. von der Ecken, R. Ahrens, W. Freude, A. E. Guber, C. Koos, Lab Chip, 17, 1740-1748 (2017).

[135] A. Ashkin, J. M. Dziedzic, Physical Review Letters, 38, 1351-1354 (1977).

[136] S. Avino, A. Krause, R. Zullo, A. Giorgini, P. Malara, P. De Natale, H. P. Loock, G. Gagliardi, Advanced Optical Materials, 2, 1155-1159 (2014).

[137] S. Maayani, L. L. Martin, T. Carmon, Nat Commun, 7, 10435 (2016).

[138] S. K. Tang, Derda, R., Quan, Q., Lončar, M. and Whitesides, G.M., Optics express, 19, 2204-2215 (2011).

[139] V. D. Ta, R. Chen, H. D. Sun, Sci Rep, 3, 1362 (2013).

[140] T. A. Kumar, M. A. Mohiddon, N. Dutta, N. K. Viswanathan, S. Dhara, Applied Physics Letters, 106, (2015).

[141] S. Yang, V. D. Ta, Y. Wang, R. Chen, T. He, H. V. Demir, H. Sun, Scientific Reports, 6, (2016).

[142] M. Saito, T. Hashimoto, J. Taniguchi, Opt Lett, 42, 4119-4122 (2017).

[143] A. Jonáš, Z. Pilát, J. Ježek, S. Bernatová, T. Fořt, P. Zemánek, M. Aas, A. Kiraz, Journal of the Optical Society of America B, 34, (2017).

[144] S. Kaminski, L. L. Martin, T. Carmon, Optics Express, 23, (2015).

[145] S. Anand, M. Eryürek, Y. Karadag, A. Erten, A. Serpengüzel, A. Jonáš, A. Kiraz, Journal of the Optical Society of America B, 33, (2016).

[146] Y. Karadag, M. Aas, A. Jonas, S. Anand, D. McGloin, A. Kiraz, Opt Lett, 38, 16691671 (2013).

[147] Z. Liu, L. Liu, Z. Zhu, Y. Zhang, Y. Wei, X. Zhang, E. Zhao, Y. Zhang, J. Yang, L. Yuan, Opt Lett, 41, 4649-4652 (2016).

[148] L. Zheng, M. Zhi, Y. Chan, S. A. Khan, Lab Chip, 18, 197-205 (2017).

[149] L. J. Chen, L. L. Gong, Y. L. Lin, X. Y. Jin, H. Y. Li, S. S. Li, K. J. Che, Z. P. Cai, C. J. Yang, Lab Chip, 16, 1206-1213 (2016).

[150] K. Han, K. Zhu, G. Bahl, Applied Physics Letters, 105, (2014). 
[151] Y. Yang, Y. Ooka, R. M. Thompson, J. M. Ward, S. N. Chormaic, Opt Lett, 41, 575$578(2016)$.

[152] S. H. Huang, S. Sheth, E. Jain, X. Jiang, S. P. Zustiak, L. Yang, Opt Express, 26, 51$62(2018)$.

[153] M. Sumetsky, Dulashko, Y. and Windeler, R.S., Optics letters, 35, 898-900 (2010).

[154] A. Watkins, Ward, J., Wu, Y. and Chormaic, S.N., Optics letters, 36, 2113-2115

(2011).

[155] G. Wang, X. Liu, Z. Gui, Y. An, J. Gu, M. Zhang, L. Yan, G. Wang, Z. Wang, Sensors (Basel), 17, (2017).

[156] R. Henze, Ward, J.M. and Benson, O., Optics express, 21, 675-680 (2013).

[157] Z. Zhou, F. Shu, Z. Shen, C. Dong, G. Guo, Science China Physics, Mechanics \& Astronomy, 58, (2015).

[158] M. Sumetsky, Dulashko, Y. and Windeler, R.S., Optics letters, 35, 1866-1868 (2010).

[159] Z. H. Zhou, C. L. Zou, Y. Chen, Z. Shen, G. C. Guo, C. H. Dong, Opt Express, 25, 4046-4053 (2017).

[160] R. Henze, Seifert, T., Ward, J. and Benson, O., Optics letters, 36, 4536-4538 (2011).

[161] Y. Yang, S. Saurabh, J. Ward, S. N. Chormaic, Opt Lett, 40, 1834-1837 (2015).

[162] Y. Yang, F. Lei, S. Kasumie, L. Xu, J. M. Ward, L. Yang, S. Nic Chormaic, Opt Express, 25, 1308-1313 (2017).

[163] Y. Yang, J. Ward, S. N. Chormaic, Opt Express, 22, 6881-6898 (2014).

[164] J. M. Ward, Y. Yang, S. N. Chormaic, IEEE Photonics Technology Letters, 25, 23502353 (2013).

[165] G. Bahl, K. H. Kim, W. Lee, J. Liu, X. Fan, T. Carmon, Nat Commun, 4, 1994 (2013).

[166] T. Tang, X. Wu, L. Liu, L. Xu, Appl Opt, 55, 395-399 (2016).

[167] G. Persichetti, I. A. Grimaldi, G. Testa, R. Bernini, Sensors and Actuators A: Physical, 280, 271-276 (2018).

[168] T. Reynolds, N. Riesen, A. Meldrum, X. Fan, J. M. M. Hall, T. M. Monro, A.

François, Laser \& Photonics Reviews, 11, (2017).

[169] J. M. Ward, Y. Yang, F. Lei, X.-C. Yu, Y.-F. Xiao, S. N. Chormaic, Optica, 5, (2018).

[170] H. Jing, H. Lü, S. K. Özdemir, T. Carmon, F. Nori, Optica, 5, (2018).

[171] X. Tu, S.-L. Chen, C. Song, T. Huang, L. J. Guo, IEEE Photonics Journal, 11, 1-10 (2019).

[172] S. M. Grist, S. A. Schmidt, J. Flueckiger, V. Donzella, W. Shi, S. Talebi Fard, J. T.

Kirk, D. M. Ratner, K. C. Cheung, L. Chrostowski, Optics Express, 21, 7994-8006 (2013).

[173] Z. Guo, Y. Qin, P. Chen, J. Hu, Y. Zhou, X. Zhao, Z. Liu, Y. Fei, X. Jiang, X. Wu,

Small, 16, (2020).

[174] S. F. Wondimu, M. Hippler, C. Hussal, A. Hofmann, S. Krammer, J. Lahann, H. Kalt,

W. Freude, C. Koos, Opt Express, 26, 3161-3173 (2018).

[175] T. Reynolds, A. Francois, N. Riesen, M. E. Turvey, S. J. Nicholls, P. Hoffmann, T. M. Monro, Anal Chem, 88, 4036-4040 (2016).

[176] A. Francois, T. Reynolds, T. M. Monro, Sensors (Basel), 15, 1168-1181 (2015).

[177] M. Charlebois, A. Paquet, L. S. Verret, K. Boissinot, M. Boissinot, M. G. Bergeron, C. N. Allen, Nanoscale Res Lett, 5, 524-532 (2010).

[178] M. E. Anderson, E. C. O'Brien, E. N. Grayek, J. K. Hermansen, H. K. Hunt,

Biosensors (Basel), 5, 562-576 (2015).

[179] E. Ozgur, P. Toren, O. Aktas, E. Huseyinoglu, M. Bayindir, Sci Rep, 5, 13173 (2015).

[180] S. Mahalingam, Z. Xu, M. Edirisinghe, Langmuir, 31, 9771-9780 (2015).

[181] L. Fu, Q. Lu, X. Liu, X. Chen, X. Wu, S. Xie, Talanta, 213, 120815 (2020).

[182] X. Zhang, L. Liu, L. Xu, Applied Physics Letters, 104, (2014).

[183] K. Han, J. Kim, G. Bahl, Optica, 3, (2016).

[184] J. Suh, K. Han, C. W. Peterson, G. Bahl, APL Photonics, 2, (2017). 
[185] H. Ghali, H. Chibli, J. L. Nadeau, P. Bianucci, Y. A. Peter, Biosensors (Basel), 6, 20 (2016).

[186] A. J. Qavi, R. C. Bailey, Angew Chem Int Ed Engl, 49, 4608-4611 (2010).

[187] Z. Ballard, M. D. Baaske, F. Vollmer, Sensors, 15, 8968-8980 (2015).

[188] F. Vollmer, D. Braun, A. Libchaber, M. Khoshsima, I. Teraoka, S. Arnold, Applied Physics Letters, 80, 4057-4059 (2002).

[189] A. L. Washburn, W. W. Shia, K. A. Lenkeit, S.-H. Lee, R. C. Bailey, Analyst, 141, 5358-5365 (2016).

[190] F. Arduini, S. Cinti, V. Scognamiglio, D. Moscone, G. Palleschi, Anal Chim Acta, 959, 15-42 (2017).

[191] Y. J. Chen, U. Schoeler, C. B. Huang, F. Vollmer, Small, 14, e1703705 (2018).

[192] B.-B. Li, Q.-Y. Wang, Y.-F. Xiao, X.-F. Jiang, Y. Li, L. Xiao, Q. Gong, Applied

Physics Letters, 96, (2010).

[193] C. H. Dong, L. He, Y. F. Xiao, V. R. Gaddam, S. K. Ozdemir, Z. F. Han, G. C. Guo, L. Yang, Applied Physics Letters, 94, (2009).

[194] B. Özel, R. Nett, T. Weigel, G. Schweiger, A. Ostendorf, Measurement Science and Technology, 21, (2010).

[195] C. He, H. Sun, J. Mo, C. Yang, G. Feng, H. Zhou, S. Zhou, Laser Physics, 28, (2018).

[196] L. L. Martín, C. Pérez-Rodríguez, P. Haro-González, I. R. Martín, 19, 25792-25798 (2011).

[197] D. V. Strekalov, R. J. Thompson, L. M. Baumgartel, I. S. Grudinin, N. Yu, Optics express, 19, 14495-14501 (2011).

[198] J. Yu, E. Lewis, G. Brambilla, P. Wang, Sensors (Basel), 18, (2018).

[199] X. Chen, L. Fu, Q. Lu, X. Wu, S. Xie, Sensors (Basel), 18, (2018).

[200] X. Xu, W. Chen, G. Zhao, Y. Li, C. Lu, L. Yang, Light Sci Appl, 7, 62 (2018).

[201] P. Wang, M. Ding, G. S. Murugan, L. Bo, C. Guan, Y. Semenova, Q. Wu, G. Farrell, G. Brambilla, Opt Lett, 39, 5208-5211 (2014).

[202] L. de Sousa-Vieira, S. Ríos, I. R. Martín, L. García-Rodríguez, V. N. Sigaev, V. I. Savinkov, G. Yu Shakhgildyan, Optical Materials, 83, 207-211 (2018).

[203] Z. Yang, Y. Wu, X. Zhang, W. Zhang, P. Xu, S. Dai, IEEE Photonics Technology Letters, 29, 66-69 (2017).

[204] Q. Ma, L. Huang, Z. Guo, T. Rossmann, Measurement Science and Technology, 21, (2010).

[205] M. A. Md Johari, M. I. M. Abdul Khudus, M. H. Bin Jali, A. Al Noman, S. Wadi Harun, Sensors and Actuators A: Physical, 284, 286-291 (2018).

[206] L. Liang, M. Li, N. Liu, H. Sun, Q. Rong, M. Hu, Optical Fiber Technology, 45, 415418 (2018).

[207] W. X. Wei Xu, C. X. Chunxiang Xu, F. Q. Feifei Qin, Y. S. Yaqi Shan, Z. Z. Zhu Zhu, Y. Z. Ye Zhu, Chinese Optics Letters, 16, (2018).

[208] M. Eryürek, Z. Tasdemir, Y. Karadag, S. Anand, N. Kilinc, B. E. Alaca, A. Kiraz, Sensors and Actuators B: Chemical, 242, 1115-1120 (2017).

[209] M. A. Md Johari, M. I. M. Abdul Khudus, M. H. Bin Jali, A. Al Noman, S. W. Harun, Optik, 185, 558-565 (2019).

[210] L. Labrador-Paez, K. Soler-Carracedo, M. Hernandez-Rodriguez, I. R. Martin, T. Carmon, L. L. Martin, Opt Express, 25, 1165-1172 (2017).

[211] H. P. Wagner, H. Schmitzer, J. Lutti, P. Borri, W. Langbein, Journal of Applied Physics, 113, (2013).

[212] L. L. Martin, S. F. León-Luis, C. Pérez-Rodríguez, I. R. Martín, U. R. Rodríguez-

Mendoza, V. Lavín, Journal of the Optical Society of America B, 30, (2013).

[213] A. Bianchetti, A. Federico, S. Vincent, S. Subramanian, F. Vollmer, Optics

Communications, 394, 152-156 (2017). 


\section{WILEY-VCH}

[214] R. Madugani, Y. Yang, V. H. Le, J. M. Ward, S. Nic Chormaic, IEEE Photonics Technology Letters, 28, 1134-1137 (2016).

[215] M. Manzo, T. Ioppolo, Opt Lett, 40, 2257-2260 (2015).

[216] M. Manzo, T. Ioppolo, U. K. Ayaz, V. Lapenna, M. V. Otugen, Rev Sci Instrum, 83, 105003 (2012).

[217] J. Hu, S. Liu, X. Wu, L. Liu, L. Xu, Sensors (Basel), 19, (2019).

[218] X. Wang, Y. Pang, G. Ku, X. Xie, G. Stoica, L. V. Wang, Nature biotechnology, 21, 803-806 (2003).

[219] A. Rosenthal, S. Kellnberger, D. Bozhko, A. Chekkoury, M. Omar, D. Razansky, V. Ntziachristos, Laser \& Photonics Reviews, 8, 450-457 (2014).

[220] G. Wissmeyer, M. A. Pleitez, A. Rosenthal, V. Ntziachristos, Light: Science \& Applications, 7, (2018).

[221] A. Maxwell, S. W. Huang, T. Ling, J. S. Kim, S. Ashkenazi, L. J. Guo, IEEE J Sel Top Quantum Electron, 14, 191-197 (2008).

[222] T. Ling, S. L. Chen, L. J. Guo, Appl Phys Lett, 98, 204103 (2011).

[223] S. M. Leinders, W. J. Westerveld, J. Pozo, P. L. van Neer, B. Snyder, P. O'Brien, H. P. Urbach, N. de Jong, M. D. Verweij, Sci Rep, 5, 14328 (2015).

[224] H. Li, B. Dong, Z. Zhang, H. F. Zhang, C. Sun, Sci Rep, 4, 4496 (2014).

[225] B. Dong, H. Li, Z. Zhang, K. Zhang, S. Chen, C. Sun, H. F. Zhang, Optica, 2, (2015).

[226] J. Pan, B. Zhang, Z. Liu, J. Zhao, Y. Feng, L. Wan, Z. Li, Photonics Research, 8, (2020).

[227] G. Frigenti, L. Cavigli, A. Fernández-Bienes, F. Ratto, S. Centi, T. García-Fernández, G. Nunzi Conti, S. Soria, Physical Review Applied, 12, (2019).

[228] R. M. J. Murphy, F. Lei, J. M. Ward, Y. Yang, S. N. Chormaic, Optics Express, 25, (2017).

[229] E. Rubino, T. Ioppolo, Appl Opt, 56, 229-233 (2017).

[230] R. Schilling, H. Schütz, A. H. Ghadimi, V. Sudhir, D. J. Wilson, T. J. Kippenberg,

Physical Review Applied, 5, (2016).

[231] S. Lane, J. Chan, T. Thiessen, A. Meldrum, Sensors and Actuators B: Chemical, 190, 752-759 (2014).

[232] F. Sedlmeir, R. Zeltner, G. Leuchs, H. G. L. Schwefel, Optics Express, 22, (2014).

[233] S. Tang, Y. Fang, Z. Liu, L. Zhou, Y. Mei, Lab on a Chip, 16, 182-187 (2016).

[234] A. R. Ali, T. Ioppolo, V. Ötügen, M. Christensen, D. MacFarlane, Journal of Polymer Science Part B: Polymer Physics, 52, 276-279 (2014).

[235] A. R. Ali, M. A. Kamel, Mathematical Problems in Engineering, 2017, 1-8 (2017).

[236] T. Ioppolo, E. Rubino, Journal of Polymer Science Part B: Polymer Physics, 52, 272275 (2014).

[237] A. Mahmood, V. Kavungal, S. S. Ahmed, G. Farrell, Y. Semenova, Optics Letters, 40, (2015).

[238] A. Mahmood, V. Kavungal, S. S. Ahmed, P. Kopcansky, V. Zavisova, G. Farrell, Y. Semenova, Opt Express, 25, 12195-12202 (2017).

[239] J. Zhu, G. Zhao, I. Savukov, L. Yang, Scientific Reports, 7, (2017).

[240] S. Zhu, L. Shi, N. Liu, X. Xu, X. Zhang, Microfluidics and Nanofluidics, 21, (2017).

[241] M. Agarwal, I. Teraoka, Anal Chem, 87, 10600-10604 (2015).

[242] M. Gao, C. Wei, X. Lin, Y. Liu, F. Hu, Y. S. Zhao, Chem Commun (Camb), 53, 31023105 (2017).

[243] B. Yao, C. Yu, Y. Wu, S.-W. Huang, H. Wu, Y. Gong, Y. Chen, Y. Li, C. W. Wong, X. Fan, Y. Rao, Nano Letters, 17, 4996-5002 (2017).

[244] V. D. Ta, R. Chen, D. M. Nguyen, H. D. Sun, Applied Physics Letters, 102, (2013). 